\title{
Risk Management for a Global Supply Chain Planning under Uncertainty: Models and Algorithms
}

\author{
Fengqi You ${ }^{1}$, John M. Wassick ${ }^{2}$, Ignacio E. Grossmann ${ }^{1 *}$ \\ ${ }^{1}$ Dept. of Chemical Engineering, Carnegie Mellon University, Pittsburgh, PA 15213 \\ ${ }^{2}$ The Dow Chemical Company, Midland, MI 48674
}

July 2008

\begin{abstract}
In this paper we consider the risk management for mid-term planning of a global multi-product chemical supply chain under demand and freight rate uncertainty. A two-stage linear stochastic programming approach is proposed within a multi-period planning model that takes into account the production and inventory levels, transportation modes, times of shipments and customer service levels. To investigate the potential improvement by using stochastic programming, we describe a simulation framework that relies on a rolling horizon approach. The studies suggest that at least $5 \%$ savings in the total real cost can be achieved compared to the deterministic case. In addition, an algorithm based on the multi-cut L-shaped method is proposed to effectively solve the resulting large scale industrial size problems. We also introduce risk management models into the stochastic programming model, and multi-objective optimization schemes are implemented to establish the tradeoffs between cost and risk. To demonstrate the effectiveness of the proposed stochastic models and decomposition algorithms, a case study of a realistic global chemical supply chain problem is presented.
\end{abstract}

Keywords: Supply Chain Management, Risk Management, Stochastic Programming, Multicut L-shaped Method, Simulation

\footnotetext{
* To whom all correspondence should be addressed. Email: grossmann@cmu.edu; Tel.: +1 412268 3642; fax: +1 4122687139 .
} 


\section{Introduction}

Global supply chains in the process industries are usually very large scale systems that can be comprised of up to hundreds of or even thousands of production facilities, distribution centers and customers. Due to competition in the global marketplace, process industries are facing increasing pressure to manage their supply chains so as to reduce costs and risks. ${ }^{1,2}$ To achieve this goal, effective mathematical tools for large-scale supply chain optimization, particularly for cost reduction and risk management, have drawn significant attention. ${ }^{3}$

This paper is motivated by a real world application originating at The Dow Chemical Company, which has several global business units that supply multiple products to world wide customers. A large business unit can spend well into the hundreds of millions of dollars every year on its supply chain to handle and distribute the products. A deterministic planning model can be a useful tool to help the business units reduce costs by taking into account the flexibility in the system to shift production, inventory, and shipping volumes in such a way that customer demand is met while costs are minimized. However, due to inaccurate forecasts of customer demands and energy prices, supply chain planning gives rise to various types of financial risks. Since addressing these problems is a non-trivial task, it is the objective of this work to develop optimization models and solution algorithms for the risk management of large scale supply chain tactical planning under demand and freight rate uncertainties.

We consider in this paper the problem of midterm planning for a large scale multiproduct supply chain under demand and freight rate uncertainty for which a two-stage stochastic linear programming approach is proposed, incorporating a multi-period planning model that takes into account the production and inventory levels, transportation modes, times of shipments and customer service levels. In the two-stage framework, the production, distribution and inventory decisions for the current time period are made "here-and-now" prior to the resolution of uncertainty, while the decisions for the rest time periods are postponed in a "wait-and-see" mode. A resulting challenge is that a large number of scenarios are required because the problem includes a very large number of uncertain parameters due to the multi-period nature and the large size of the supply chain network. To reduce the model size and the number of scenarios, we use a Monte Carlo sampling approach to discretize the continuous probability distribution functions and to generate the scenarios. To quantify the cost saving 
achieved by modeling uncertainty in supply chain planning, we describe a simulation framework that relies on a "rolling horizon" approach. Simulation studies on the case problem suggest that at least 5\% saving in the total cost can be achieved by using the stochastic approach compared to the deterministic one. To solve the resulting large scale industrial size problems effectively, an algorithm based on the multi-cut L-shaped method is proposed. As an additional enhancement, we introduce four risk management models by incorporating different risk measures into the proposed stochastic programming model. Different risk metrics, including variance, variability index, probabilistic financial risk and downside risk are used to explicitly measure the risks arising from uncertain customer demands and freight rates which allow managing these risks according to the decision maker's preference. Multi-objective optimization schemes are also developed to tradeoff the cost minimization and risk minimization objectives for the global supply chain planning. To demonstrate the effectiveness of the proposed stochastic models and decomposition algorithms, a realistic case study of a global chemical supply chain problem is presented.

The problem addressed in this paper has a number of novel features. First, we incorporate Monte Carlo sampling in a stochastic programming framework to reduce the number of scenarios for a real world application. Secondly, we propose a simulation framework based on iteratively solving deterministic and stochastic programming problems so as to quantitatively assess the cost savings achieved by the use of stochastic programming. The third feature is that we take into account the selection of different transportation modes with different transportation times in the stochastic programming model and the simulation framework. To our knowledge, planning problems that consider transportation time and transportation modes under uncertainty have not been addressed in this manner. An additional feature is that we implemented a multi-cut L-shaped method to solve the large scale problem from a real world case study. The proposed algorithm proved to be very effective for solving large-scale stochastic linear programming problems. Moreover, we present a comprehensively comparison of several risk management models for planning under uncertainty.

The rest of this paper is organized as follows. Section 2 reviews some relevant literature on supply chain tactical planning under uncertainty and risk management. The general problem statement is given in Section 3. Section 4 presents the two-stage stochastic programming model. A simulation framework to quantify the different costs by using the stochastic and deterministic approaches is proposed in Section 5. An 
efficient algorithm to solve the industrial size problems is presented in Section 6. Section 7 presents the risk management models, along with a comparison between different risk measures and the solution quality. Numerical results from a real world case study of a large-scale global chemical supply chain problem are presented in Section 8. Finally, Section 9 concludes on the performance of the proposed stochastic models and decomposition algorithms.

\section{Literature Review}

Tactical supply chain planning typically covers a midterm time horizon of between few months to one year, and decisions cover issues such as production, inventory and distribution. ${ }^{4}$ Related work includes, for instance, the one by Wilkinson et al., ${ }^{5}$ who propose an approach to integrate production and distribution in multisite facilities using the resource task network framework. Bok et al. ${ }^{6}$ propose a multiperiod supply chain optimization model for operational planning of continuous flexible process networks where sales, intermittent deliveries, production shortfalls, delivery delays, inventory profiles and changeovers costs are taken into account. A bilevel decomposition algorithm was proposed, which reduced the computational time significantly. Jackson and Grossmann ${ }^{7}$ present a temporal decomposition scheme based on Lagrangean decomposition for a nonlinear programming problem model for multi-site production and distribution planning, where nonlinear terms arise from the relationship between production and physical properties or blending ratios. Chen et al. ${ }^{8}$ present a multi-product, multistage and multiperiod production and distribution planning model. They also proposed a two-phase fuzzy decision making method to obtain a compromise solution among all participants of the multi-enterprise supply chain. A multiproduct supply chain planning model with consideration of duty drawback is proposed by Oh and Karimi. ${ }^{9}$ Recently, Guillen et al. ${ }^{10}$ present a mixed-integer linear programming model for tactical planning and operational scheduling of chemical supply chains with multi-product, multi-echelon distribution networks with consideration of financial management issues. All of these models are deterministic supply chain planning models that do not take into account the uncertainties or risks in the supply chain planning process.

A number of approaches have been proposed in the chemical engineering literature for the quantitative treatment of uncertainty in the design, planning and scheduling 
problems. A classification of different areas of uncertainty for batch chemical plant design is suggested by Subrahmanyam et al., ${ }^{11}$ where uncertainty in prices and demand, equipment reliability and manufacturing are taken into account. The authors used a scenario-based approach, which attempts to capture uncertainty by representing it in terms of a number of discrete realizations of the stochastic quantities, constituting distinct scenarios. The objective is to find a solution that performs well on average under all scenarios. The scenario-based approach provides a straightforward way to implicitly account for uncertainty. Its major drawback is that the problem size increases exponentially as the number of scenarios increases. This is particularly true when using continuous multivariate probability distribution with Gaussian quadrature integration schemes. These difficulties can sometimes be circumvented by analytically integrating continuous probability distribution functions for the random parameters. ${ }^{12,13}$ While this approach can lead to a reasonable size deterministic equivalent representation of the probabilistic model, this is often at the expense of introducing nonlinearities into the model. Furthermore, the nonlinear terms in the resulting deterministic equivalent problems are often nonconvex requiring global optimization techniques. ${ }^{14-17}$ A recent popular method to address the uncertainty is to use Monte Carlo sampling in the scenario planning framework ${ }^{18,19}$ and then combine it with statistical methods to determinate the number of required scenarios so as to achieve a desired level of accuracy. ${ }^{20}$ By using this method, the required number of scenarios in the stochastic program can be significantly reduced, while the solution quality can be guaranteed at the desired level. ${ }^{21}$ In this work, we use the Monte Carlo sampling method to deal with large scale supply chain planning problem under uncertainty.

In the stochastic programming models, the total expected performance measure is optimized so as to obtain optimal solutions that perform well on average for all the scenarios. However, standard stochastic programming methods usually do not provide any control on the solution's variability over the different scenarios. In other words, the decision makers are assumed to be risk-neutral. One may have different attitudes towards the risk, thus the supply chain risks should be controlled and managed based on the decision makers' preference. Related works about risk management includes, for instance, Eppen and Martin ${ }^{22}$ who propose the downside risk as a risk measure and incorporated it into a two-stage stochastic programming model for the production capacity planning under demand uncertainty in auto industry. Later Mulvey et al. ${ }^{23}$ describe a robust optimization model to control the mean value and variance of the 
objective functions in stochastic programs. Ahmed and Sahinidis ${ }^{24}$ propose the upper partial mean as a measure of risk and apply it in the long-term chemical process planning. Applequist et al. ${ }^{25}$ discuss risk premium as a measure that provides the basis for a rational balance between expected value of investment performance and variance. Recently, Barbaro and Bagajewicz ${ }^{26}$ introduced the probabilistic financial risk as a metric of risk for planning under uncertainty problems. Similar techniques are presented by Bonfill et al. ${ }^{27}$ for managing financial risk in scheduling problems. The probabilistic financial risk measure is also used for refinery planning ${ }^{28}$ and short term scheduling with pricing policies ${ }^{29}$.

\section{Problem Statement}

The problem addressed in this paper can be stated as follows. We are given a midterm planning horizon (for instance, one year), which can be subdivided into a number of time periods (for instance, one month as a time period). A set of products are manufactured and distributed through a given global supply chain that includes a large number of world wide customers and a number of geographically distributed plants and distribution centers. All the facilities (plants and distribution centers) can hold inventory and are connected to each other by an associated transportation link. Each customer is served by one or more facilities with specified transportation links. A simplified version of the network is shown in Figure 1. The network has multiple echelons whereby material may flow from the manufacturing plant through several distribution centers on its way to the final customer. Freight rates are specific to the transportation link involved and depend on distance and mode of transport. Generally, the transportation links are classified into two types, one is from a facility to another facility (plant or distribution center), and the other one is from a facility to a customer.

Besides the supply chain network topology, we are also given the minimum and initial inventory of each facility. The inventory holding costs and the facility throughput costs are already known, together with future monthly demand of each product by each customer. The transportation time of each shipping lane is known and should be taken into account. 


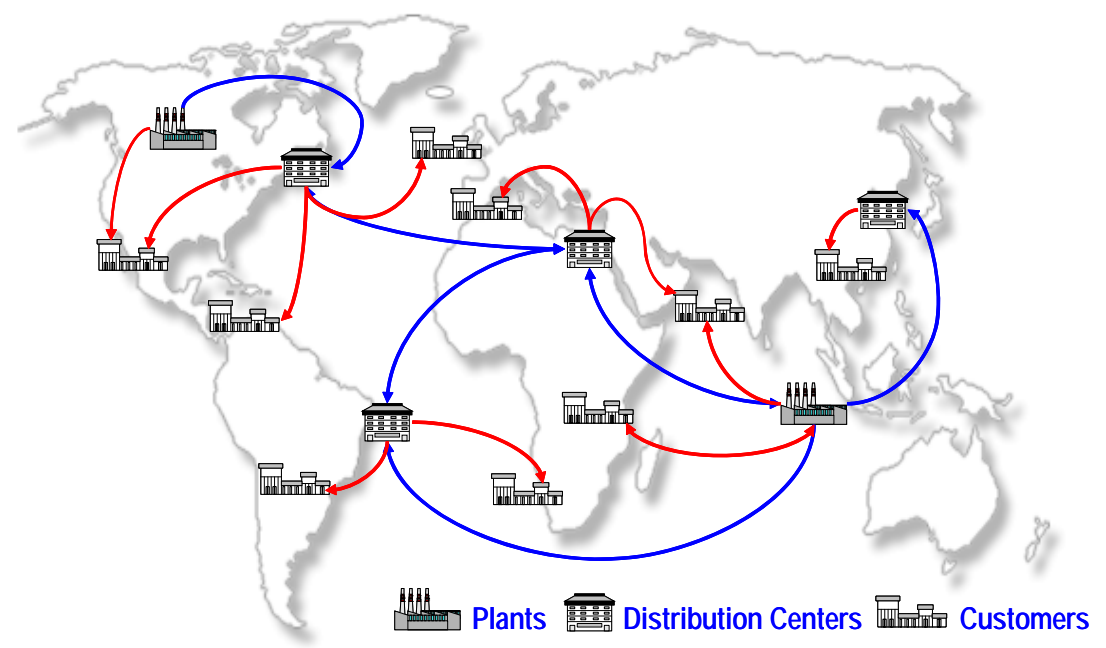

Figure 1 Global chemical supply chain

The uncertainties arise from the customer demands and freight rates. The values of these uncertain parameters follow some probability distribution (such as, but not restricted to, normal distribution) with given mean and variance. Usually, the probability distribution of the uncertain parameters can be obtained by fitting the historical data for different probability distributions, or based on expert opinions. The mean values of these uncertain parameters typically come from forecasting, and the variances come from historical data. ${ }^{12}$ It is important to note that we allow the demands and freight rates to have different levels of uncertainties changing with time. For example, in January the uncertain demand of May has a standard deviation as much as $20 \%$ of the mean value, but in April the standard deviation of that demand of May reduces to $5 \%$ of the mean value due to more accurate forecasting and information. Different levels of uncertainties are very important for the operations of industrial supply chains, and should be taken into account in the models.

The problem is to determine the monthly production ${ }^{\mathrm{I}}$ and inventory levels of each facility, and the monthly shipping quantities between network nodes such that the total expected cost and the total risks of the global supply chain are minimized while satisfying customer demands over the specified planning horizon.

\footnotetext{
${ }^{\mathrm{I}}$ The model could be also easily extended to deal with weekly production planning (or even shorter time interval) by changing the length of time periods.
} 


\section{Stochastic Programming Model}

\subsection{Two-stage Approach}

We consider a two-stage stochastic programming ${ }^{30}$ approach to deal with different levels of uncertainties and incorporate it into a multi-period planning model that takes into account the production and inventory levels, transportation modes and times of shipments and the customer service levels. ${ }^{\text {II }}$ In the two-stage framework, the production, distribution and inventory decisions for the current time period are made "here-and-now" prior to the resolution of uncertainty, while the decisions for the rest of the time periods are postponed in a "wait-and-see" mode after the uncertainties are revealed. The scenario planning approach is used to represent the uncertainties. A resulting challenge is that a large number of scenarios are required because the problem includes a very large number of uncertain parameters due to the multi-period nature of the model and the large size of global supply chain network. To reduce the model size and the number of scenarios, we use a Monte Carlo sampling approach to generate the scenarios. Each scenario is then assigned the same probability with the summation of the probabilities for all the scenarios equal to $1 .{ }^{18,19}$ For example, if we use Monte Carlo sampling to generate 100 scenarios, the probability of each scenario is given as 0.01 . The number of scenarios is determined by using a statistical method ${ }^{20,21}$ to obtain solutions within specific confidence intervals for a desired level of accuracy. This method is very effective for scenario reduction, particularly for large-scale problems. As an example, for a problem with $5^{1000}$ scenarios, a sample size of around 400 can find the true optimal solution with probability $95 \%{ }^{20}$

\subsection{Mathematical Formulation}

In this work, we use a multi-period formulation to allow the costs and sourcing decisions to change with time while taking into account the transportation time for each shipment. The model includes five types of constraints. They are mass balance constraints for the production plants, distribution centers and customers, together with the constraints for production capacity and minimum inventory levels. The definition of sets, variables and parameters of the model are given at the end of this paper. Note that exchange rates, taxes, tariffs and duty drawbacks use linear approximations ${ }^{9,31}$ and

\footnotetext{
II In principle, the problem can be formulated as a multi-stage stochastic programming model, to reduce the computational efforts we only consider a two-stage approach.
} 
are taken into account in the parameters of freight rates and facility throughput costs. The mathematical formulation of the multi-period linear programming planning model is given in the following sections.

\subsubsection{Mass balance for plants}

Let us consider the mass balance for plant $k$ product $j$ at the first time period $(t=1)$. At the first time period $(t=1)$, all the decisions are assumed to be independent of the future scenarios. The mass balance for the plant $k \in K_{P}$ product $j$ at the first time period $(t=1)$ is then given as follows.

$$
\begin{array}{r}
\sum_{k^{\prime} \in K} \sum_{m \in M} F_{k, k^{\prime}, j, m, t}+\sum_{r \in R} \sum_{m \in M} S_{k, r, j, m, t}=I_{k, j}^{0}-I_{k, j, t}+W_{k, j, t}+\sum_{k^{\prime} \in K} \sum_{m \in M} F_{k^{\prime}, k, j, m, t-\lambda_{k^{\prime}, k, j, m}}, \\
\forall j, k \in K_{P}, t=1
\end{array}
$$

Equation (1) states that the total freight shipped from plant $k \in K_{P}$ to other facilities and customers with all the transportation modes $m \in M$ should be equal to the changes in inventory plus the production amount and the volume shipped to plant $k \in K_{P}$ from other facilities. Because we need to consider the transportation time during the shipping process, the input freight coming from other facilities should start at the time period of $t-\lambda_{k^{\prime}, k, j, m}$ so that the freight can arrive at the destination at time period $t$, where $\lambda_{k^{\prime}, k, j, m}$ is the shipping time from facility $k^{\prime}$ to plant $k \in K_{P}$ of product $j$ with transportation mode $m$.

For the remaining time periods $(t \geq 2)$ contained in the second stage time periods, most of the decisions will be the second stage decisions. So for plant $k \in K_{P}$ product $j$ at time period $t \geq 2$ for scenario $s$, the mass balance can be expressed as follows.

$$
\begin{gathered}
\sum_{k^{\prime} \in K} \sum_{m \in M} F_{k, k^{\prime}, j, m, t, s}+\sum_{r \in R} \sum_{m \in M} S_{k, r, j, m, t, s}=I_{k, j, t-1, s}-I_{k, j, t, s}+W_{k, j, t, s}+\sum_{k^{\prime} \in K} \sum_{m \in M} F_{k^{\prime}, k, j, m, t-\lambda_{k^{\prime}, k, j, m}, s}, \\
\forall j, s, k \in K_{P}, t \geq 2
\end{gathered}
$$

Equation (2) is similar to Eq. (1), but all the variables are replaced by the second stage variables, i.e. related to scenario $s$. It is important to note that for the second time period ( $t=2$ ), the term $I_{k, j, t-1, s}$ in Eq. (2) refers to the ending inventory level of the first time period, which is a first stage decision independent of scenarios. Similarly, if the freight $F_{k^{\prime}, k, j, m, t-\lambda_{k^{\prime}, k, j, m}, s}$ starts from the first time period, i.e. $t=\lambda_{k^{\prime}, k, j, m}+1$, then the freight is also a first stage decision independent of the scenario. 


\subsubsection{Mass balance for distribution centers}

For the distribution centers, the mass balance equation is very similar to that for a plant; only the production term $W_{i, j, t}$ is missing. So for distribution center $k \in K_{D C}$ and product $j$ in the first time period $(t=1)$, the mass balance equation is given by:

$$
\begin{aligned}
\sum_{k^{\prime} \in K} \sum_{m \in M} F_{k, k^{\prime}, j, m, t}+\sum_{r \in R} \sum_{m \in M} S_{k, r, j, m, t}=I_{k, j}^{0}-I_{k, j, t}+\sum_{k^{\prime} \in K} \sum_{m \in M} F_{k^{\prime}, k, j, m, t-\lambda_{k^{\prime}, k, j, m}} & \\
\forall j, k \in K_{D C}, t & \quad t=1
\end{aligned}
$$

For the remaining time periods $(t \geq 2)$ and scenario $s$, the mass balance is given by:

$$
\begin{array}{r}
\sum_{k^{\prime} \in K} \sum_{m \in M} F_{k, k^{\prime}, j, m, t, s}+\sum_{r \in R} \sum_{m \in M} S_{k, r, j, m, t, s}=I_{k, j, t-1, s}-I_{k, j, t, s}+\sum_{k^{\prime} \in K} \sum_{m \in M} F_{k^{\prime}, k, j, m, t-\lambda_{k^{\prime}, k, j, m}, s} \\
\forall j, s, k \in K_{D C}, t \geq 2
\end{array}
$$

Similarly, for the second time period $(t=2)$, the term $I_{k, j, t-1, s}$ in Equation (4) refers to the ending inventory level of the first time period, which is a first stage decision. Thus $I_{k, j, t-1, s}$ should be replaced by $I_{k, j, t-1}$ when $t=2$. Similarly, shipments $F_{k^{\prime}, k, j, m, t-\lambda_{k^{\prime}, k, j, m}, s}$ that originate in the first time period, i.e. $t=\lambda_{k^{\prime}, k, j, m}+1$, are first stage decisions independent of the scenarios and should be replaced by $F_{k^{\prime}, k, j, m, t-\lambda_{k^{\prime}, k, j, m}}$.

\subsubsection{Mass balance for customers}

To satisfy the demand of product $j$ at customer $r$ in time period $t$, the sum of all shipments from other facilities (plants and distribution centers) via all the shipping modes $m$ starting at time period $t-\lambda_{k, r, j, m}$ (and arriving at customer $r$ at time period $t$ ) should be no less than the demand $\left(d_{r, j, t, s}\right)$. To satisfy certain service levels and to ensure the constraint is feasible, we introduce a positive slack variable $S F_{r, j, t, s}$ to quantify the unmet demand. Hence, the mass balance for product $j$ at customer $r$ in the first time period $(t=1)$ and scenario $s$ can be formulated as follows:

$$
\sum_{k \in K} \sum_{m \in M} S_{k, r, j, m, t-\lambda_{k, r, j, m}}+S F_{r, j, t, s} \geq d_{r, j, t, s}, \forall r, j, s, t=1
$$

For the remaining time periods $(t \geq 2)$ and scenario $s$, the mass balance for product $j$ at customer $r$ is given as:

$$
\sum_{k \in K} \sum_{m \in M} S_{k, r, j, m, t-\lambda_{k, r, j, m}, s}+S F_{r, j, t, s} \geq d_{r, j, t, s}, \forall r, j, s, t \geq 2
$$

Note that in constraint (6), we consider customer demand as the lower bound of the sales. One could also enforce the sale to be equal to the demand by changing constraint 
(6) as an equality.

\subsubsection{Capacity constraints}

The production amount $\left(W_{k, j, t}, W_{k, j, t, s}\right)$ of each plant $\left(k \in K_{P}\right)$ should not exceed the capacity $\left(Q_{k, j}, k \in K_{P}\right)$.

$W_{k, j, t} \leq Q_{k, j}, \forall j, t=1, k \in K_{P}$

$W_{k, j, t, s} \leq Q_{k, j}, \forall j, s, t \geq 2, k \in K_{P}$

\subsubsection{Minimum inventory constraints}

The minimum inventory of product $j$ in facility $k$ at each time period $t$ should be satisfied. Equation (9) and (10) model this constraint.

$$
\begin{aligned}
& I_{k, j, t} \geq I_{k, j, t}^{m}, \quad \forall k, j, t=1 \\
& I_{k, j, t, s} \geq I_{k, j, t}^{m}, \quad \forall k, j, s, t \geq 2
\end{aligned}
$$

\subsubsection{Objective function: expected total cost}

The objective function of this stochastic linear programming model is to minimize the total expected cost that includes the first stage cost, Cost 1 , plus the expected second stage cost. Since the scenarios follow discrete distribution, the expected second stage cost is equal to the product of the scenario probability, $p_{s}$, and the associated second stage scenario cost, Cost $2_{s}$, summed over all the scenarios $s$.

$E[\operatorname{Cos} t]=\operatorname{Cost} 1+\sum_{s \in S} p_{s} \cdot \operatorname{Cost} 2_{s}$

Both the first stage cost and the second stage scenario costs are equal to the sum of the following items:

- Inventory holding cost for all products at all facilities for all time periods

- Freight cost for inter-facility freight shipments in all the shipping lanes of all the products in all time periods

- Freight cost for facility-customer shipments in all the shipping lanes of all the products in all the time periods

- Facility throughput cost for inter-facility shipments for all the shipping lanes of all the products in all the time periods

- Facility throughput cost for facility-customer shipments for all the shipping lanes of all the products in all the first stage time periods 
- Penalty costs of all the products for lost unmet demand of all the customers in all the time periods

Thus, the first stage cost is given as,

$$
\begin{aligned}
& \text { Cost } 1=\sum_{k \in K} \sum_{j \in J} \sum_{t=1} h_{k, j, t} I_{k, j, t}+\sum_{k \in K} \sum_{k^{\prime} \in K} \sum_{j \in J} \sum_{m \in M} \sum_{t=1} \gamma_{k, k^{\prime}, j, m, t} F_{k, k^{\prime}, j, m, t}+\sum_{k \in K} \sum_{r \in R} \sum_{j \in J} \sum_{m \in M} \sum_{t=1} \gamma_{k, r, j, m, t} S_{k, r, j, m, t} \\
& +\sum_{k \in K} \sum_{k^{\prime} \in K} \sum_{j \in J} \sum_{m \in M} \sum_{t=1} \delta_{k, j, t} F_{k, k^{\prime}, j, m, t}+\sum_{k \in K} \sum_{r \in R} \sum_{j \in J} \sum_{m \in M} \sum_{t=1} \delta_{k, j, t} S_{k, r, j, m, t}
\end{aligned}
$$

The cost of each scenario $s$ is equal to,

$$
\begin{gathered}
\operatorname{Cost}_{s}=\sum_{k \in K} \sum_{j \in J} \sum_{t \geq 2} h_{k, j, t} I_{k, j, t, s}+\sum_{k \in K} \sum_{k^{\prime} \in K} \sum_{j \in J} \sum_{m \in M} \sum_{t \geq 2} \gamma_{k, k^{\prime}, j, m, t, s} F_{k, k^{\prime}, j, m, t, s}+\sum_{k \in K} \sum_{r \in R} \sum_{j \in J} \sum_{m \in M} \sum_{t \geq 2} \gamma_{k, r, j, m, t, s} S_{k, r, j, m, t, s} \\
+\sum_{k \in K} \sum_{k^{\prime} \in K} \sum_{j \in J} \sum_{m \in M} \sum_{t \geq 2} \delta_{k, j, t} F_{k, k^{\prime}, j, m, t, s}+\sum_{k \in K} \sum_{r \in R} \sum_{j \in J} \sum_{m \in M} \sum_{t \geq 2} \delta_{k, j, t} S_{k, r, j, m, t, s}+\sum_{r \in R} \sum_{j \in J} \sum_{t \in T} \eta_{r, j, t} S F_{r, j, t, s} \\
, \forall S
\end{gathered}
$$

Minimizing the objective function in (11) - (13), subject to the constraints in (1) (10), we can obtain the solution for the two-stage stochastic programming model. However, since the number of scenarios may be too large we use a sampling scheme as discussed in the next section.

\subsection{Calculation of Confidence Interval}

The number of scenarios is determined by the desired level of accuracy of the solution, which can be measured by the confidence interval of the expected total cost. The confidence interval can be calculated as follows. The Monte Carlo sampling variance estimator of the result for a stochastic programming problem, which is independent of the probability distribution of the uncertain parameters, is given by, ${ }^{18,19}$

$$
S(n)=\sqrt{\frac{\sum_{s=1}^{n}\left(E[\text { Cost }]-\text { Cost }_{s}\right)^{2}}{n-1}}
$$

where $n$ is the number of scenarios and Cost $_{s}$ is the total cost of scenarios.

Then the confidence interval of $1-\alpha$ is given as:

$$
\left[E[\text { Cost }]-\frac{z_{\alpha / 2} S(n)}{\sqrt{n}}, E[\text { Cost }]+\frac{z_{\alpha / 2} S(n)}{\sqrt{n}}\right]
$$

where $z_{\alpha / 2}$ is the standard normal deviate such that $1-\alpha / 2$ satisfies for a standard normal distributed variable $z \sim N(0,1), \operatorname{Pr}\left(z \leq z_{\alpha / 2}\right)=1-\alpha / 2$. For example, for $95 \%$ confidence interval (i.e. $1-\alpha=95 \%$ ), we have $z_{\alpha / 2}=1.96$. 
On the other hand, if we are given the sampling estimator $S(n)$ and the desired confidence interval $H$, the minimum number of scenarios required can be determined by,

$N=\left[\frac{z_{\alpha / 2} S(n)}{H}\right]^{2}$

Therefore, to determine the number of scenarios $N$, we first solve the stochastic programming model with a small number of scenarios $n$ (such as 10-100), to estimate the value of sampling estimator $S(n)$ by using Eq. (14). Then using Eq. (15) and (16), we can determine the required number of scenarios for a desired confidence interval. ${ }^{20}$, 21

\section{Simulation Framework}

To assess the impact of using the stochastic programming approach, we developed a simulation framework. The basic idea is to compare the simulated operations of two planners, one using a deterministic model for planning and the other one using a stochastic model for planning (Figure 2). At the beginning of each time period, the stochastic planner will run the two-stage stochastic programming model with the current time period (for instance, month) as the first stage time period in the model and the remaining time periods as the second stage time period. After a solution is returned, the stochastic planner will execute the decisions for the current time period. Similar actions will be taken by the deterministic planner using the deterministic model. After both planners execute their decisions, the system randomly generates the information for demands and freight rates. These include the realization of the uncertain demand for the current time period, and the forecasting values of demands and freight rates for the future time periods. Both planners then update their information. The stochastic planner uses the information for both mean values and variances of the uncertain parameters (including the demands and freight rates), while the deterministic planner only uses the mean values of the uncertain parameters. Once the information is updated both planners move on to the next time period.

The entire system operates under a rolling horizon approach as shown in Figure 3. For example, if the planning horizon is 12 months and January is the current time period, the stochastic planner will run the stochastic model with the decisions for January as the first stage decisions and the decisions for this February to December as 
the second stage decisions. After the problem is solved, the planner will execute the decisions for January only. Then, in the next iteration the decisions for February are considered as the first stage decisions, and the decisions for this March to the following January are treated as the second stage decisions. The process continues until decisions are executed for December. The deterministic planner follows a similar process using the deterministic model and mean values of the uncertain parameters.

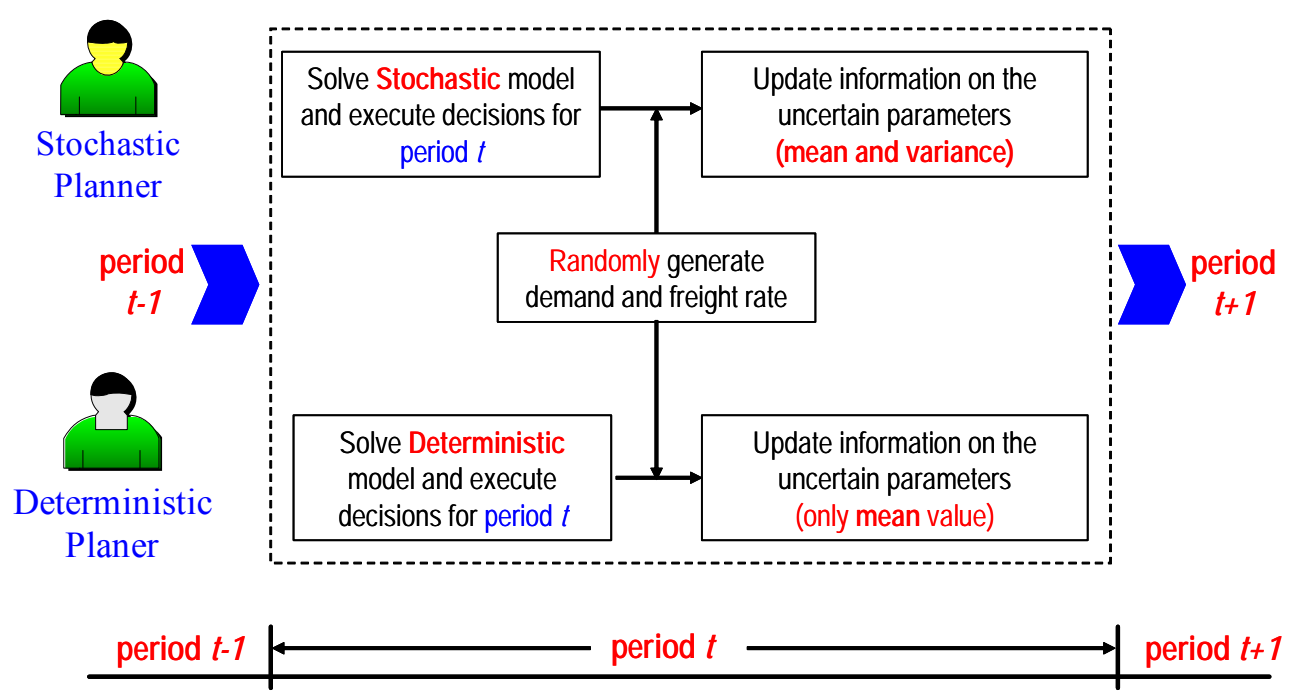

Figure 2 Simulation framework

This process gives rise to a rolling horizon where the first time period of the model is moving forward but the length of the planning horizon is unchanged. The process once initiated continues until an entire year's decisions have been made. In this way we simulate the typical planning cycle carried on in an industrial setting.

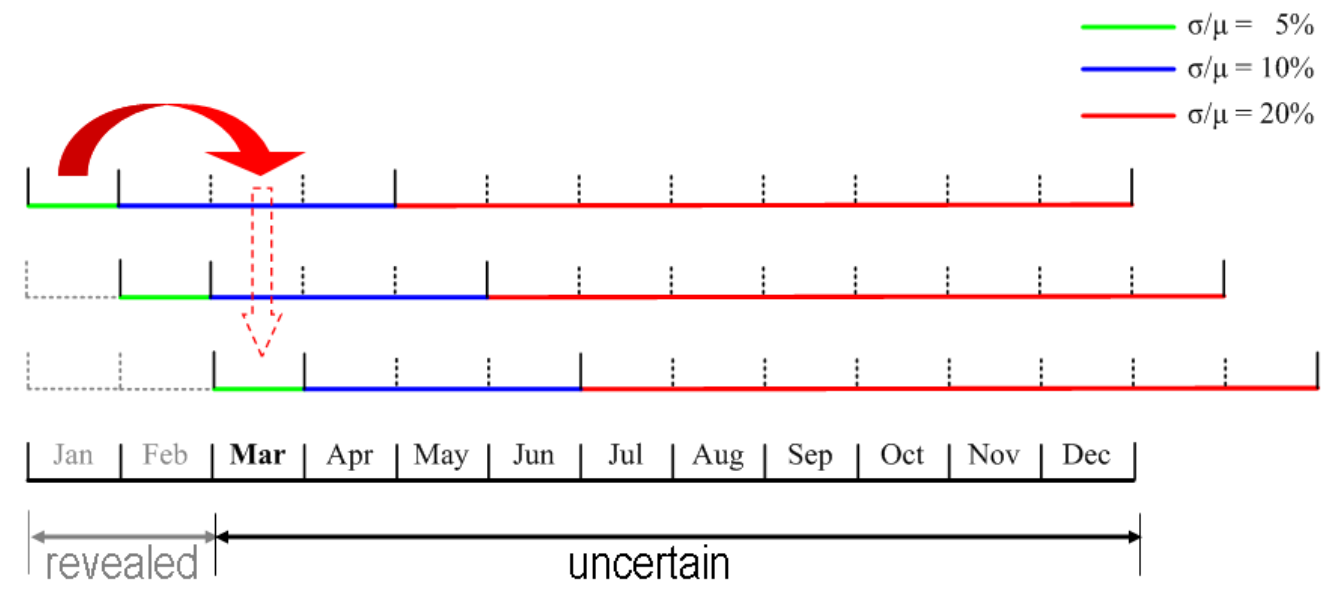




\section{Figure 3 Rolling horizon strategy}

There are several issues that require special attention in this simulation framework. The first is initial conditions. The previous time period's ending inventory represents the initial inventory of the new time period. For example, in the second iteration (for the decisions of February), the ending inventory of January should be treated as the initial inventory of February.

Another issue we need to take into account is the transportation time. All inter-facility shipments initiated in previous time periods should be treated as pipeline inventories and considered as part of the initial inventories at the destinations in the arrival time periods. For example, if in the first iteration, the planner decides to ship some product from one facility to another and the shipment takes two time periods to arrive, then the amount of this shipment should be considered as part of the initial inventory of the destination for March in the next two iterations, i.e. the second time period in the second iteration and the first time period in the third iteration (Figure 3). Similarly, for a shipment from a facility to a customer, the amount of the shipment should be considered as part of the demand realization in future time periods if the transportation time exceeds one time period.

The third issue is the difference in demand and freight rate uncertainty depending on the length of the forecast. As the rolling horizon moves forward, the variance of the uncertain demand for a particular time period will change because we consider different levels of uncertainty for different forecasting horizons. This must be taken into account in the simulation. For example in Figure 3, the demand for March has a standard deviation of $10 \%$ of the mean value in the first and second iterations, but in the third iteration, March becomes the first time period and the standard deviation of demand is reduced to $5 \%$ of the mean value.

A final issue that must be dealt with is the variation in the results that are obtained for a simulated year due to the random customer demand driving the optimization. In other words, the difference between the stochastic case and the deterministic will vary in different simulation cycles because the demands encountered may be different. To address this variation the simulation system iterates through a selected number of simulation cycles to produce data that can be used to report statistics of the difference between the annual performance of the stochastic case and the deterministic case. This process gives rise to an inner loop stepping though the time periods (for instance, 
months) and an outer loop iterating through simulation cycles (for instance, years). A flow chart of the whole simulation framework is shown in Figure 4.

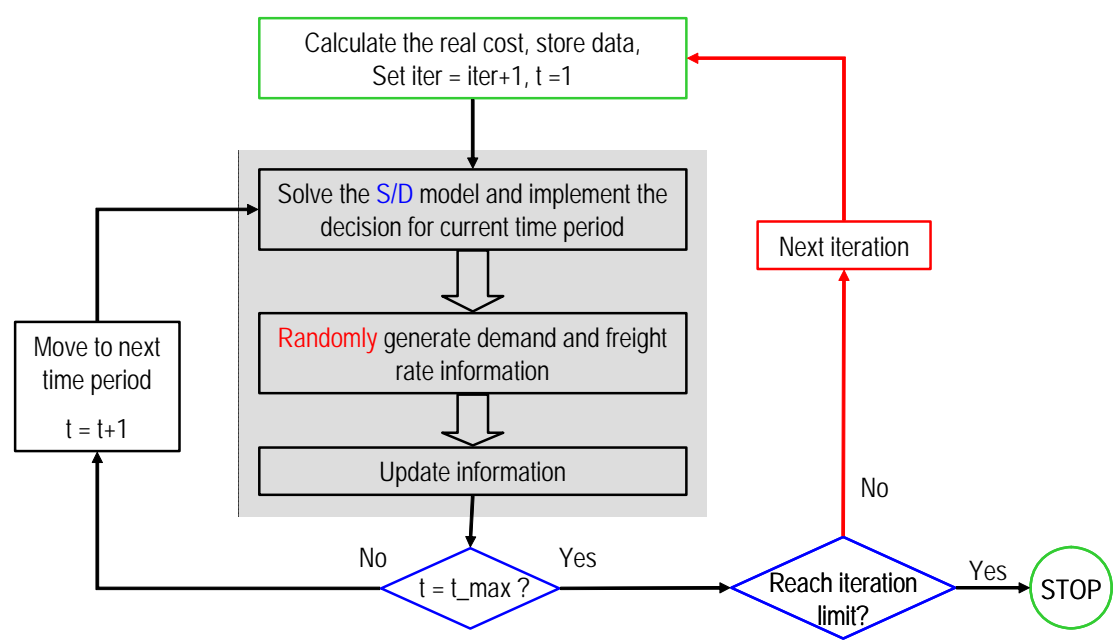

Figure 4 Simulation flowchart

\section{Solution Algorithm}

Stochastic programming models that rely on scenarios are often computationally very demanding because their model size increases exponentially as the number of scenarios increases. In particular, the deterministic equivalent of the problem addressed in this paper cannot be solved directly due to its very large size (see Section 8 for details). Therefore, we need an effective algorithm to overcome the computational challenges. A popular method for solving stochastic programming models is the L-shaped method, ${ }^{30,32}$ which takes advantage of the special decomposable structure of the two-stage stochastic programming model. Consider the following general form of the two-stage stochastic programming model (P0).

$$
\begin{array}{ll}
\min _{x, y_{s}} & c^{\mathrm{T}} x+\sum_{s \in S} p_{s} q_{s}{ }^{\mathrm{T}} y_{s} \\
\text { s.t. } & A x=b, \quad x>0 \\
& W y_{s}=h_{s}-T_{s} x, \quad y\left(w_{s}\right) \geq 0, s \in S
\end{array}
$$

where $x$ is the vector that stands for the first stage decision variables, and $y_{s}$ are the second stage decisions for each scenario $s$. Equation (17) stands for the objective function given in (11)-(13). Equation (18) stands for the constraints without second stage decisions, i.e. constraint (1), (3), (7) and (9) in the stochastic programming model. 
Equation (19) is for the second stage constraints, i.e. constraint (2), (4), (5), (6), (8), (10) in the stochastic programming model. $c$ and $q_{s}$ are the vector of coefficients for the first and second stage decisions in the objective function, i.e. the unit inventory, shipping, throughput, and penalty costs. $A$ and $b$ are parameter matrix independent of the scenarios, while $W, h_{s}$ and $T_{s}$ are parameter matrix for each scenario $s \in S$.

The expanded version of the general model (P0) is given in equation (20). We can see that the model has a special "angular" form, which can be decomposed into a master problem and a number of scenario subproblems.

$$
\begin{aligned}
& \operatorname{Min} c^{T} x+p_{1} q_{1}^{T} y_{1}+p_{2} q_{2}^{T} y_{2} \cdot \cdot \cdot+p_{s} q_{s}^{T} y_{s} \\
& \text { s.t. } A x \quad=b \rightarrow \text { Master problem } \\
& \begin{array}{ll}
T_{1} x+W_{1} y_{1} & =h_{1}
\end{array} \\
& T_{2} x+W_{2} y_{2} \quad=h_{2}
\end{aligned}
$$

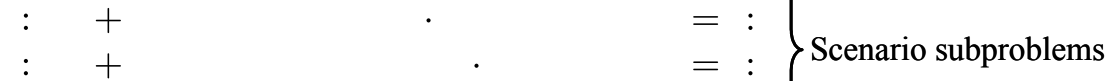

$$
\begin{aligned}
& \begin{array}{rrrr}
: & + & & \\
: & + & &
\end{array} \\
& T_{s} x+\quad W_{s} y_{s}=h_{s} \\
& x \geq 0, y_{1} \geq 0, y_{2} \geq 0, \cdots y_{s} \geq 0
\end{aligned}
$$

The basic idea of the standard L-shaped method is to first solve the model with those constraints that do not include the second stage variables to obtain the values of first stage decisions. Then we fix the first stage decisions and solve all the scenario sub-problems that include second stage decisions to obtain the optimal values of the second stage decisions.

If we define $Q_{s}(x)$ as the objective function value of each scenario subproblem $s$, $Q_{s}(x)=\min _{y_{s}} q_{s}^{\mathrm{T}} y_{s}$

$$
\text { s.t. } W y_{s}=h_{s}-T_{s} x, y\left(w_{s}\right) \geq 0
$$

then the reformulation of (P0) is as follows,

$$
\begin{array}{ll}
\min _{x} & c^{\mathrm{T}} x+\sum_{s \in S} p_{s} Q_{s}(x) \\
\text { s.t. } & A x=b, \quad x>0
\end{array}
$$

To solve (P0), we can take advantage of the dual properties of (21) by introducing a new variable $\theta$ for $\sum_{s \in S} p_{s} Q_{s}(x)$, and iterate between the master problem $(\mathbf{P 1})$ and the 
scenario subproblems (P2).

The master problem $(\mathbf{P 1})$ is given by,

(P1)

$$
\begin{array}{ll}
\min _{x, \theta} & c^{\mathrm{T}} x+\theta \\
\text { s.t. } & \theta \geq e_{l} x+d_{l}, \quad l=1 . . N \\
& A x=b, \quad x>0
\end{array}
$$

while the subproblem (P2) for scenario $s$ is given by,

$$
\begin{array}{ll}
\min _{y_{s}} & q_{s}^{\mathrm{T}} y_{s} \\
\text { s.t. } & W y_{s}=h_{s}-T_{s} x, \quad y\left(w_{s}\right) \geq 0
\end{array}
$$

where the inequalities in (P1) are the "cuts" that link the master problem and the scenario subproblems. $e_{l}$ and $d_{l}$ are coefficients for the Benders cut, and they are given by,

$e_{l}=\sum_{s \in S} p_{s} \pi_{s}^{T} T_{s}$

$d_{l}=\sum_{s \in S} p_{s} \pi_{s}^{T} h_{s}$

where $\pi_{s}$ are the optimal dual vectors of constraint (21) in the subproblem (P2) for scenario $s$.

The major steps for the L-shaped method are given in Figure 5. In this algorithm, we first solve the master problem to obtain a lower bound of the objective value. We then fix all the first stage decisions and solve each scenario subproblem to get an upper bound. If the lower bound and the upper bound are within a tolerance, then the algorithm stops. Otherwise, we use the duals of the scenario sub-problems to add a cut and return to the master problem. 


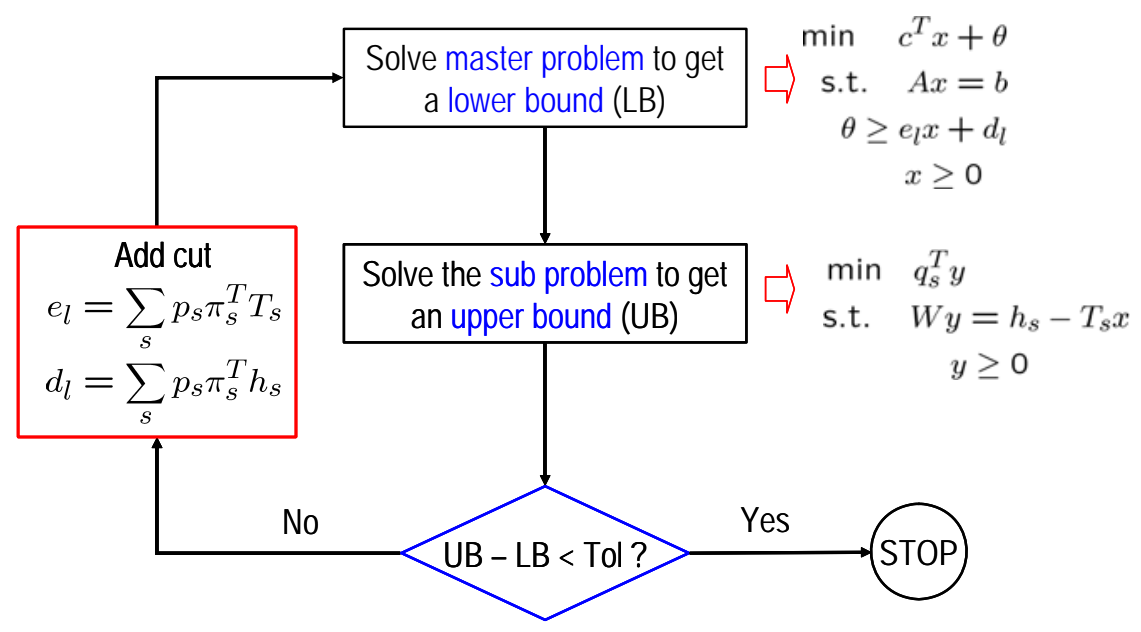

Figure 5 Algorithm for standard L-shaped method

The standard L-shaped method only returns one cut to the master problem during each iteration. In order to speed up the algorithm we can decompose the variable $\theta$ by scenario to return as many cuts as the number of scenarios in each iteration. The master problem is then given by (P3).

(P3)

$$
\begin{array}{ll}
\min _{x, \theta_{s}} & c^{\mathrm{T}} x+\sum_{s \in S} p_{s} \theta_{s} \\
\text { s.t. } & \theta_{s} \geq e_{s l} x+d_{s l}, \quad l=1 . . N \\
& A x=b, \quad x>0
\end{array}
$$

where the coefficients $e_{s l}$ and $d_{s l}$ for the cut (26) are updated as follows

$$
\begin{aligned}
& e_{s l}=p_{s} \pi_{s}^{T} T_{s} \\
& d_{s l}=p_{s} \pi_{s}^{T} h_{s}
\end{aligned}
$$

The algorithm framework for multi-cut L-shaped method is similar to the standard L-shaped method, and is given in Figure 6. 


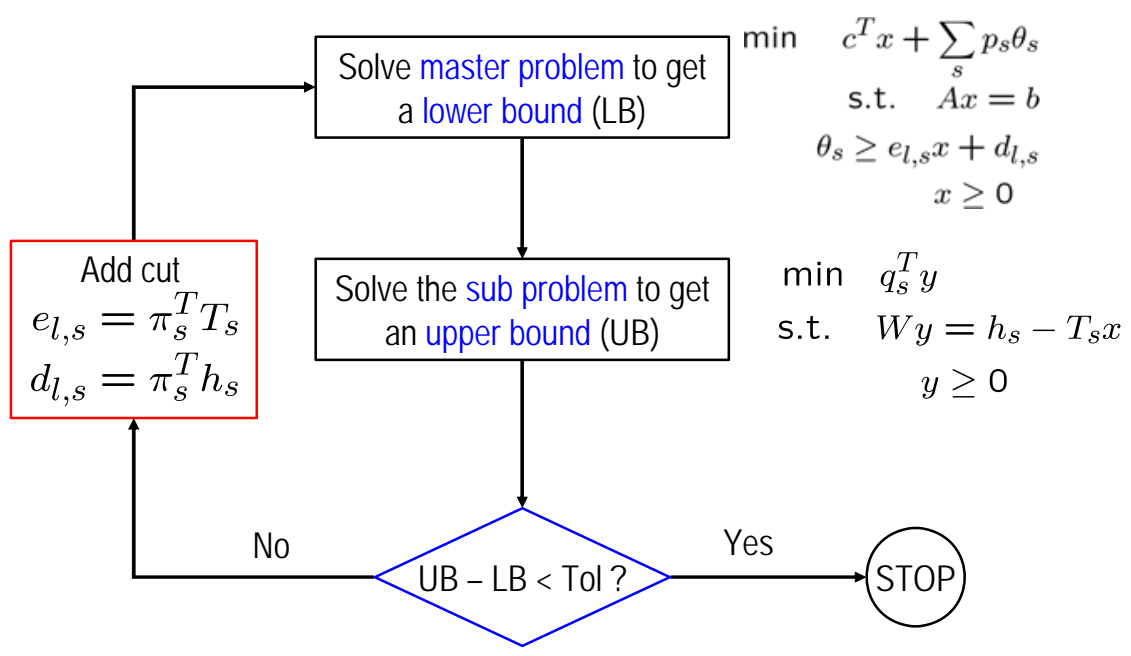

Figure 6 Algorithm for multi-cut L-shaped method

Although the multi-cut L-shaped method can provide stronger cuts to the master problem and reduce the number of iterations, it introduces more variables in the objective function of the master problem, which may potentially slow down the computation. Computational results for comparing these two algorithms are presented in Section 8. We should also note that convergence is guaranteed in both cases. ${ }^{30,32}$

\section{Risk Management Models}

In the stochastic programming model we optimize the total expected cost to obtain the optimal solutions that are optimal on average for all the scenarios. However, the expected total cost is a risk-neutral objective that cannot manage the risks explicitly. On the other hand, some decision makers are risk-averse and would like to manage the risks and improve the economic objective simultaneously. This requires extending the aforementioned stochastic programming for risk management. To manage the risk, we need first to define a metric for risks. For comparison purpose, we consider in this work four popular risk measures including variance, ${ }^{23}$ variability index, ${ }^{24}$ probabilistic financial risk $^{26}$ and downside risk. ${ }^{22}$

\subsection{Managing the Variance}

Due to the uncertain environment the total realized cost is also uncertain (see Figure 7). This cost has a mean value and a variance. Our objective in the stochastic programming model is to minimize the expected value of the total cost, while the 
variance of the total cost is not addressed. Thus, it is possible that an optimum solution may have low expected cost but a large variance. Application of such a solution would therefore involve a high amount of risk in that the possibility exists for the realized cost to be far higher than the expected value. If a decision maker is risk adverse such a solution would not be satisfactory. Therefore, we may want to find a "robust" solution that would yield similar results but also considers the variance of the solution. The risk management by variance is also called "robust optimization" in most of the Operations Research literature. ${ }^{23}$ In robust optimization, we not only minimize the expected total cost, but also minimize the variance of the total cost. Since the original stochastic programming solution is the minimum expected cost solution the solution from robust optimization usually results in higher expected cost but with less variance.

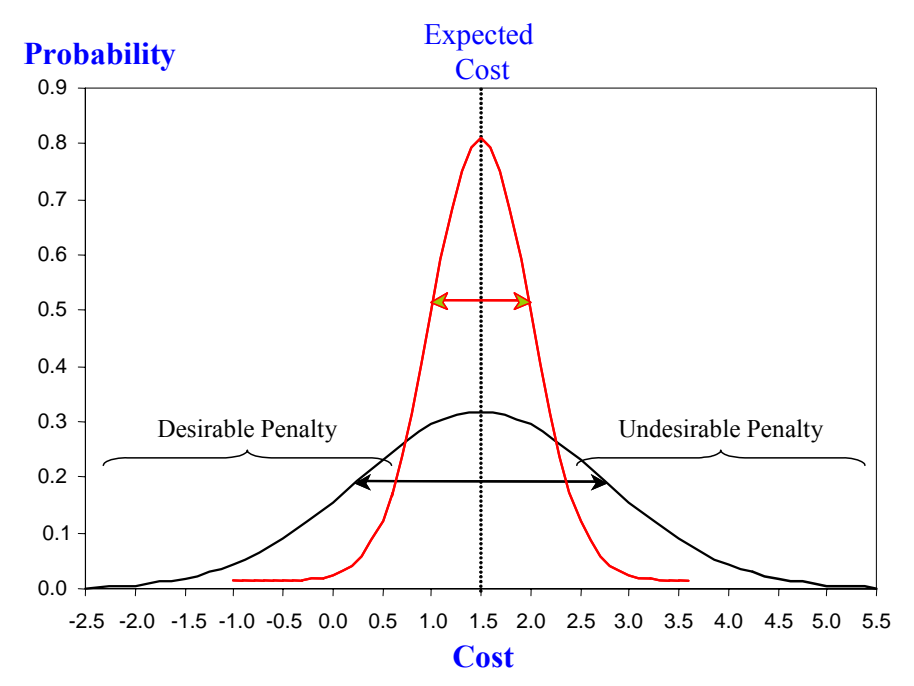

Figure 7 Robust optimization

Since risk is measured by variance, a straightforward extension to reduce its value is to add a variance term to the objective function of the stochastic program. This yields a goal programming formulation to reduce both the expected cost and the variance. The new objective function is:

$$
\begin{aligned}
\min & E[\operatorname{Cos} t]+\rho \cdot V[\operatorname{Cos} t] \\
& =\operatorname{Cos} t 1+\sum_{s \in S} p_{s} \cdot \operatorname{Cost} 2_{s}+\rho \cdot \sum_{s \in S} p_{s} \cdot\left[\left(\sum_{s^{\prime} \in S} p_{s^{\prime}} \cdot \operatorname{Cos} t 2_{s^{\prime}}\right)-\operatorname{Cos} t 2_{s}\right]^{2}
\end{aligned}
$$

where the expected cost term is equal to the first stage cost (Cost 1$)$ plus the expected second stage $\operatorname{cost}\left(\sum_{s} p_{s} \cdot \operatorname{Cos} 2_{s}\right)$. The variance term $(V[\operatorname{Cos} t])$ is equal to the mean 
square error between the expected second stage cost $\left(\sum_{s^{\prime} \in S} p_{s^{\prime}} \cdot \operatorname{Cost} 2_{s^{\prime}}\right)$ and the second stage scenario $\operatorname{cost}\left(\operatorname{Cost} 2_{s}\right)$. The coefficient $\rho$ in the objective function is the weight coefficient for the variance. For different values of $\rho$ we can tradeoff lower expected cost with lower cost variance.

In summary, the variance management model includes the objective function given in (29), (12), (13), subject to constraints (1)-(10).

\subsection{Managing the Variability Index}

The variance management model is a straightforward approach to reduce both expected value and variance of cost, but it includes a quadratic terms in the objective function (29) that makes the optimization problem difficult to solve for large scale problems. To circumvent this problem an alternative is to use the positive deviation between the scenario cost $\left(\operatorname{Cos} t 1+\operatorname{Cos} t 2_{s}\right)$ and the expected cost $\left(\operatorname{Cos} t 1+\sum_{s^{\prime}} P_{s^{\prime}} \cdot \operatorname{Cost} 2_{s^{\prime}}\right)$. Ahmed and Sahinidis ${ }^{24}$ defined the variability index (or called "upper partial mean") as a non-negative continuous variable $\Delta_{s}$ for each scenario that is defined by the following constraints:

$\Delta_{s} \geq \operatorname{Cost} 2_{s}-\left(\sum_{s^{\prime}} P_{s^{\prime}} \cdot \operatorname{Cost} 2_{s^{\prime}}\right), \Delta_{s} \geq 0, s \in S$

Equation (30) states that if the scenario cost (Cost $)$ is less than the expected cost ( $E[$ Cost $]), \Delta_{s}$ would be 0 ; If the Cost $_{s}$ is greater than the $E[$ Cost $], \Delta_{s}$ would be equal to their positive difference. This reformulation, which can be interpreted as a 1-norm measure of the variance, yields a linear programming problem which can be solved more efficiently.

The objective function of the variability index management model is to minimize the weighted sum between total expected cost and the expected variability index. Thus, the variability index management model is as follows:

$$
\begin{array}{ll}
\min & E[\text { Cost }]+\rho \cdot \sum_{s \in S} p_{s} \cdot \Delta_{s} \\
\text { s.t. } & \Delta_{s} \geq \operatorname{Cost} 2_{s}-\left(\sum_{s^{\prime}} P_{s^{\prime}} \cdot \operatorname{Cost} 2_{s^{\prime}}\right), \Delta_{s} \geq 0, s \in S \\
& \text { Constraints (1)-(10) }
\end{array}
$$

Similarly, for different values of the weighted parameter $\rho$, we can tradeoff the expected cost and the cost variability index. 


\subsection{Managing the Probabilistic Financial Risk}

Sometimes decision makers are not satisfied with a robust solution in which the variance of the cost is limited. Instead they are more concerned with the extremes of the cost spread. For example they may want a lower probability of high cost or a higher probability of low cost. In this case, we can use another risk measure, the so called probabilistic financial risk. ${ }^{26}$ This measure is defined as the probability that the real cost is higher than a certain threshold or target $\Omega$ (Figure 8). By reducing the probabilistic financial risk for threshold or target $\Omega$, we can reduce the risk of having high costs.

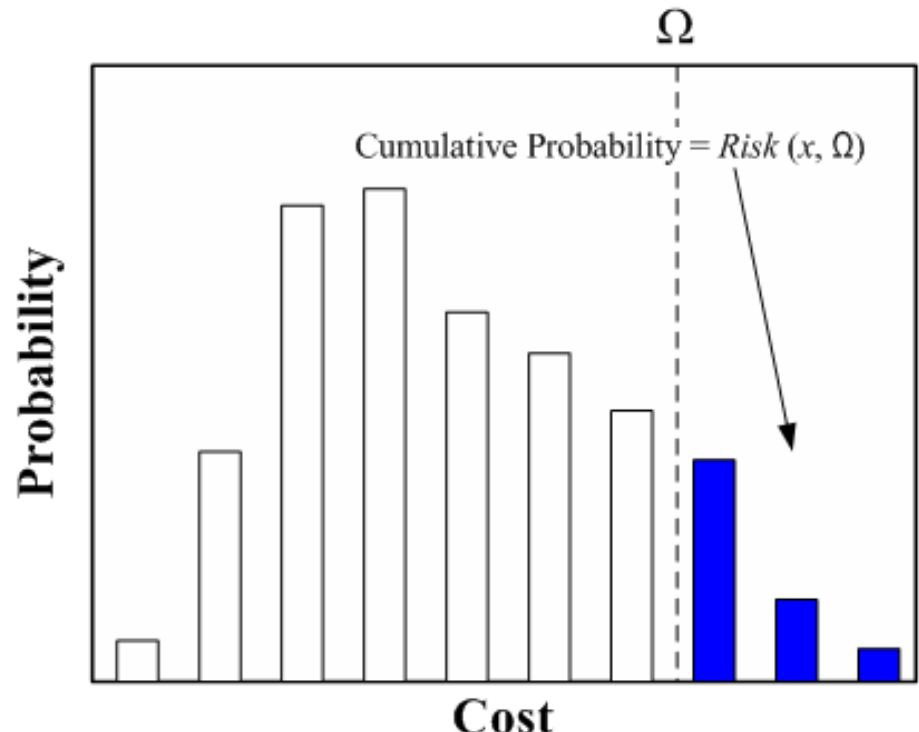

Figure 8 Probabilistic financial risk

Using the target $\Omega$, risk can be defined as the probability of the cost being greater than $\Omega$. For a scenario planning model we can introduce a binary variable $Z_{s}$, such that $Z_{s}$ equal to 1 if $\operatorname{Cost}_{s} \geq \Omega$, otherwise equal to 0 .

To define the value of the binary variables $Z_{s}$, Barbaro and Bagajewicz ${ }^{26}$ proposed the following Big-M constraints:

$\operatorname{Cost}_{s} \leq \Omega+M \cdot Z_{s}, s \in S$

$\operatorname{Cost}_{s} \geq \Omega-M \cdot\left(1-Z_{s}\right), s \in S$

where $M$ is a sufficient large positive parameter. Constraints (32) and (33) state that if 
the scenario cost Cost $_{s}$ is greater than the target $\Omega, Z_{s}$ must be 1 or else constraint (32) will be violated; if the scenario cost Cost $_{s}$ is less than the target $\Omega, Z_{s}$ must be 0 or else constraint (33) will be violated. By doing this, we define $Z_{s}$ as an indicator for each scenario.

Thus, the probabilistic financial risk is equal to the summation over all the scenarios for the product of the scenario probability and the binary variable $Z_{s}$.

$\operatorname{Risk}(x, \Omega)=\operatorname{Pr}[\operatorname{Cost}(x) \geq \Omega]=\sum_{s \in S} p_{s} \cdot Z_{s}$

The probabilistic financial risk management model is then follows:

$$
\begin{array}{ll}
\min & E[\operatorname{Cost}]=\operatorname{Cost} 1+\sum_{s \in S} p_{s} \cdot \operatorname{Cost} 2_{s} \\
\min & \operatorname{Risk}(x, \Omega)=\sum_{s \in S} p_{s} \cdot Z_{s} \\
\text { s.t. } & \text { Cost }_{s} \leq \Omega+M \cdot Z_{s}, s \in S \\
& \text { Cost }_{s} \geq \Omega-M \cdot\left(1-Z_{s}\right), s \in S
\end{array}
$$

Constraints (1)-(10)

This model has two objective functions: to minimize the probabilistic financial risk in (34) and minimize the expected total cost in (11), (12), (13), subject to the constraints (1)-(10), (32)-(33). As there are two conflicting objective functions, the corresponding problem yields an infinite set of Pareto-optimal solutions for which it is not possible to improve both objective functions simultaneously. ${ }^{34}$

In order to obtain the Pareto-optimal curve for the bi-criterion optimization problem, one of the objectives is specified as an inequality with a fixed value for the bound which is treated as a parameter. There are two major approaches to solve the problem in terms of this parameter. One is to simply solve it for a specified number of points to obtain an approximation of the Pareto optimal curve, which is the $\varepsilon$-constraint method. ${ }^{34}$ The other is to solve it as a parametric programming problem, ${ }^{35}$ which yields the exact solution for the Pareto optimal curve. While the latter provides a rigorous solution approach, the former is simpler to implement. For this reason we have selected the first approach. The procedure includes the following three steps. The first one is to minimize the expected cost $E[$ Cost $]$ to obtain the minimum expected cost, which in turn yields the largest Pareto optimal risk $\operatorname{Risk}(x, \Omega)$. The second step is to minimize $\operatorname{Risk}(x, \Omega)$ that yields the smallest Pareto optimal expected risk $\operatorname{Risk}(x, \Omega)$. The last 
step is to fix the risk $\operatorname{Risk}(x, \Omega)$ to discrete values between the smallest and greatest value, and optimize the model by minimizing $E[$ Cost $]$ at each selected point. In this way we can obtain an approximation to the Pareto-optimal curve, together with the optimal planning decisions for different values of probabilistic financial risk.

\subsection{Managing the Downside Risk}

In the aforementioned probabilistic risk management method, a binary variable is required for each scenario to calculate the probabilistic financial risk. Thus, the risk management model size will be very large as the number of scenarios increases. To avoid the integer variable, we can use downside risk ${ }^{22}$ instead of probabilistic risk for financial risk management. The basic idea is to introduce a positive deviation variable $\psi_{s}$ defined as the variability index of scenario $s$. The variable $\psi_{s}$ is defined as the positive deviation between the target $\Omega$ and the scenarios cost $\operatorname{Cost}_{s}$. If the scenario cost Cost $_{s}$ is less than the target $\Omega, \psi_{s}$ is equal to 0 . If the scenario cost Cost $_{s}$ is greater than the target $\Omega, \psi_{s}$ is equal to their difference. These conditions can be enforced with the following inequalities:

$\psi_{s} \geq \operatorname{Cost}_{s}-\Omega, \psi_{s} \geq 0, s \in S$

Then the downside risk associated with target $\Omega$, is defined as follows,

$$
\operatorname{DRisk}(x, \Omega)=\sum_{s} P_{s} \cdot \psi_{s}
$$

Thus, we have the downside risk management model as follows:

$$
\begin{array}{ll}
\min & E[\operatorname{Cos} t]=\operatorname{Cos} t+\sum_{s \in S} p_{s} \cdot \operatorname{Cost} 2_{s} \\
\min & \operatorname{DRisk}(x, \Omega)=\sum_{s} P_{s} \cdot \psi_{s} \\
\text { s.t. } & \psi_{s} \geq \text { Cost }_{s}-\Omega, \psi_{s} \geq 0, s \in S \\
& \text { Constraints (1)-(10) }
\end{array}
$$

Similar to the probabilistic financial risk management model, a downside risk management model also has two objective functions: to minimize the total expected cost in (11), (12), (13) and to minimize the downside risk in (36), subject to the constraints (1)-(10) and (35). The optimal solutions of this multi-objective optimization model also yield a Pareto curve, which can also be obtained by using the $\varepsilon$-constraint method. 


\section{Case Study}

In this section, we present a case study to demonstrate the effectiveness of the proposed models and algorithms. The problem is based on the global supply chain of a major commodity chemical producer. Some basic information about the global supply chain is discussed. The results for the stochastic programming model, simulation framework, decomposition algorithms, together with the results for different risk management models are presented and discussed. All the instances are modeled with GAMS $^{36}$ and solved with CPLEX solver on an IBM T60 laptop with an Intel Core Duo $1.83 \mathrm{GHz}$ CPU and 1GB RAM.

\subsection{Basic Information of the Case Study}

In the case study we consider a planning horizon of one year, which is subdivided into 12 time periods, i.e. one month as a time period. Two products are produced and distributed in a global supply chain, consisting of a global supply chain with 5 plants, 13 distribution centers, 121 transportation links and 46 customers. The customer demands and freight rates, which are uncertain, follow normal distributions with the forecast as the mean value and the variance coming from the historical record. The demand uncertainty has three levels of standard deviations (see Figure 3). For the current month the standard deviation of demand is $5 \%$ of the mean value, in the coming three months (i.e. $2-4$ month), the standard deviation is $10 \%$ of the mean value; for the remaining 8 months, the demand has a standard deviation of $20 \%$ of the mean value. Similarly, the freight rate has two levels of uncertainty. For the current month, the variance is 0 (i.e. deterministic case); in the remaining 11 months, the freight rate has a standard deviation of $10 \%$ of the mean value. All the other data about the supply chain, such as the unit cost coefficients, capacities, minimum inventory levels, are omitted due to confidentiality reasons.

\subsection{Results for Stochastic Programming Models and Simulation}

We solve the case study with a sampling size of 600 scenarios. The results are given in Figure 9 and Figure 10. The minimum total expected cost is $\$ 183.32 \mathrm{MM}$. The $95 \%$ confidence interval of the expected cost is given $\$ 0.37 \mathrm{MM}$ above and below this 
value, which is relatively small compared to the expected cost.

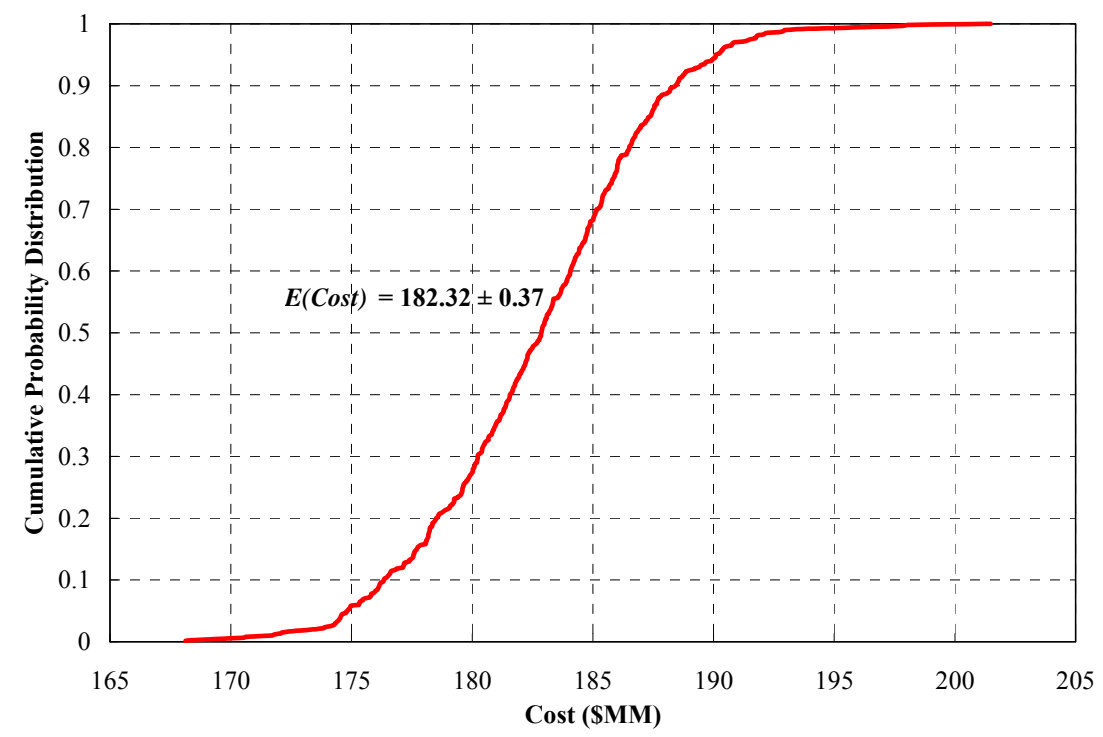

Figure 9 Cumulative probability distribution for the two-stage stochastic programming model with 600 scenarios

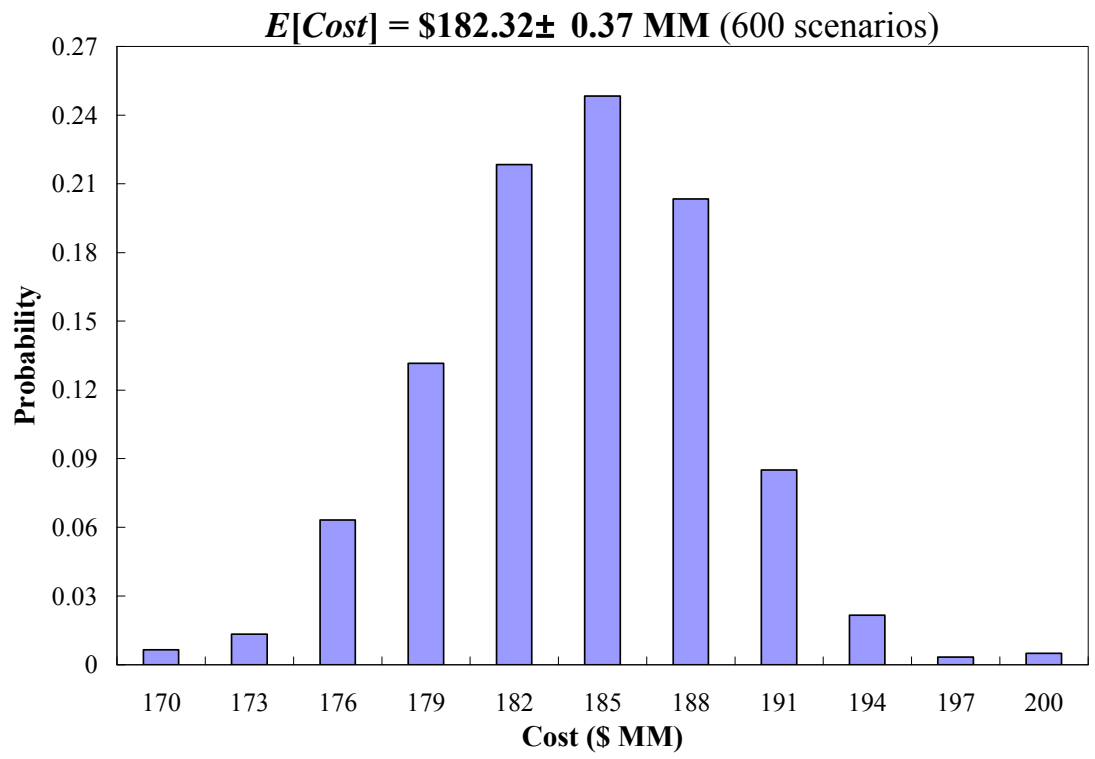

Figure 10 Histogram of the results for the two-stage stochastic programming model with 600 scenarios

To quantify the cost saving by using stochastic programming, we implement the simulation framework for the case problem. We used a sampling size of 1000 scenarios and simulated 100 iterations. Year-by-year results are given in Figure 11. The operational cost from stochastic planning is always less than the operational cost from 
deterministic planning. On average, 5.70\% cost saving was achieved by using the stochastic programming approach. Figure 12 shows the components of the average operational cost for both approaches. Figure 13 and 14 are the comparisons on the inventory levels and sourcing for one of the production facilities. As can be seen, by using the stochastic programming approach, the production facility holds less inventories compared to the one with deterministic approach (Figure 13), and thus the sourcing amounts for this facility by stochastic approach are fewer (Figure 14).

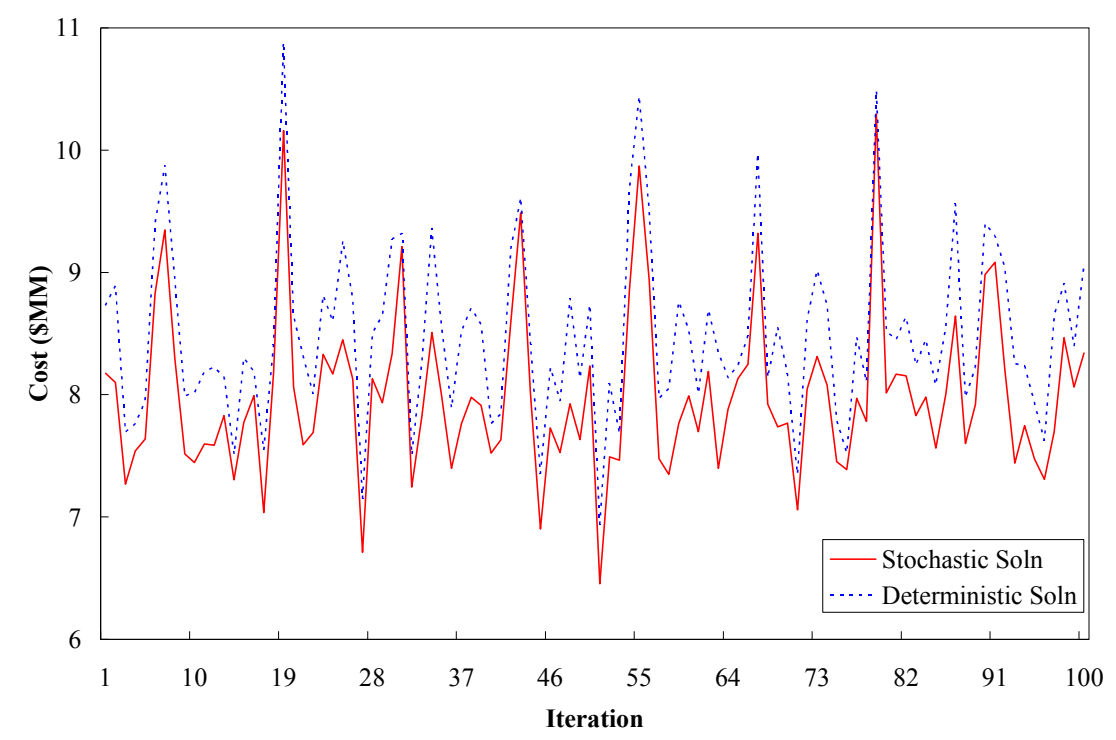

Figure 11 Simulation results for the real costs of one year planning

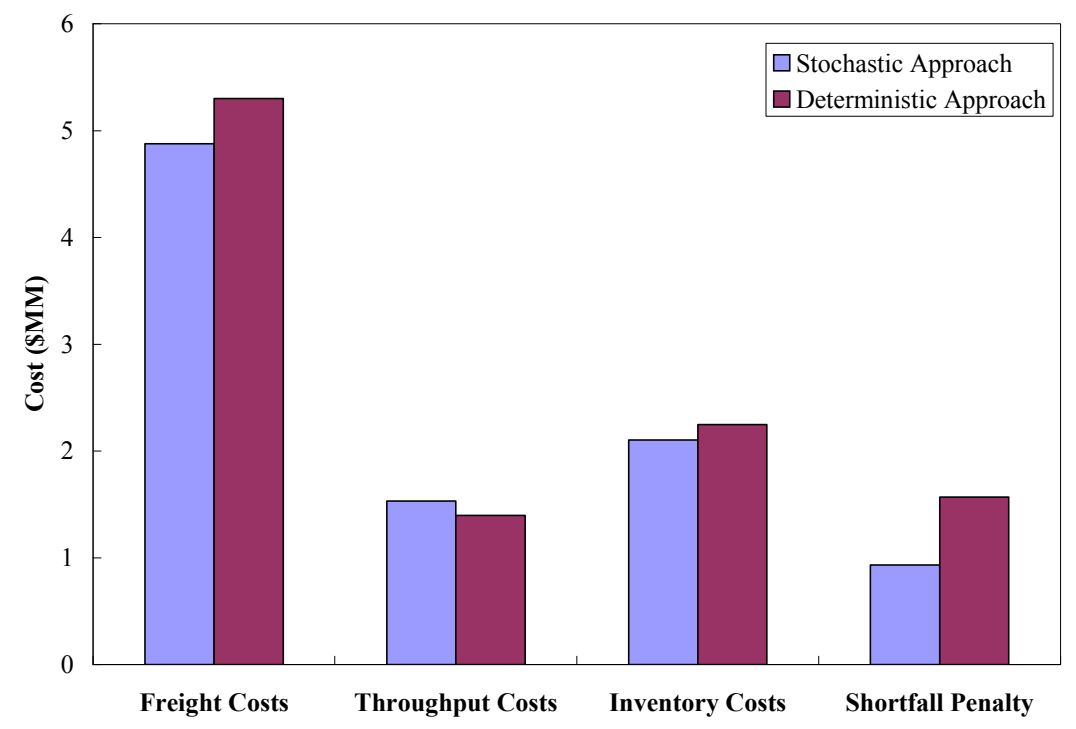

Figure 12 Average component real costs for two planners 


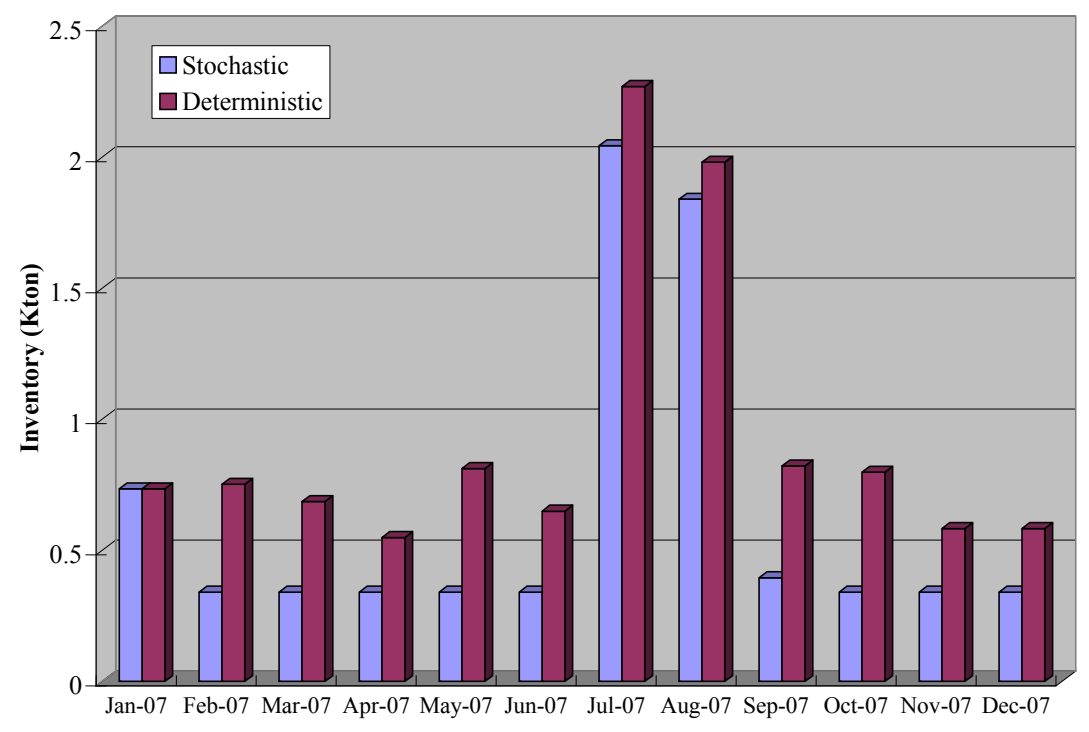

Figure 13 Comparison on inventory levels for one production facility

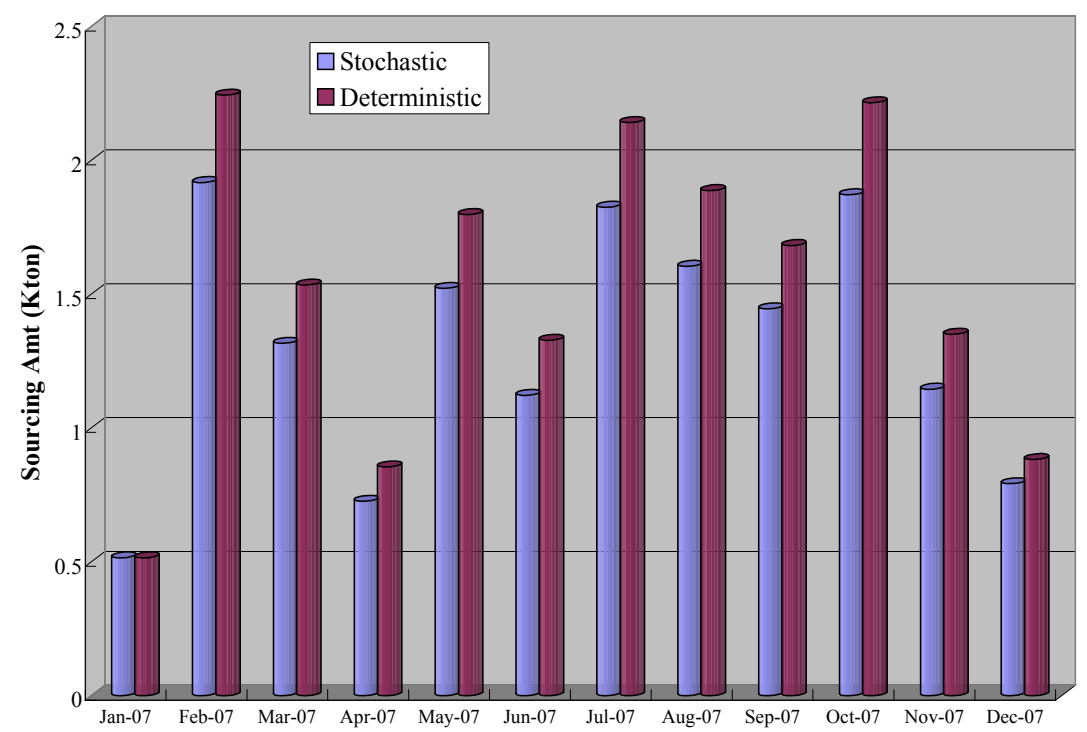

Figure 14 Comparison on sourcing amount for one production facility

As a sensitivity analysis, we doubled the uncertainty levels (increased the standard deviations of the uncertain parameters to $200 \%$ of the original setting), the results are shown in Figure 15. As can be seen, larger cost savings can be achieved by using the stochastic programming approach (on average 13.95\%). 


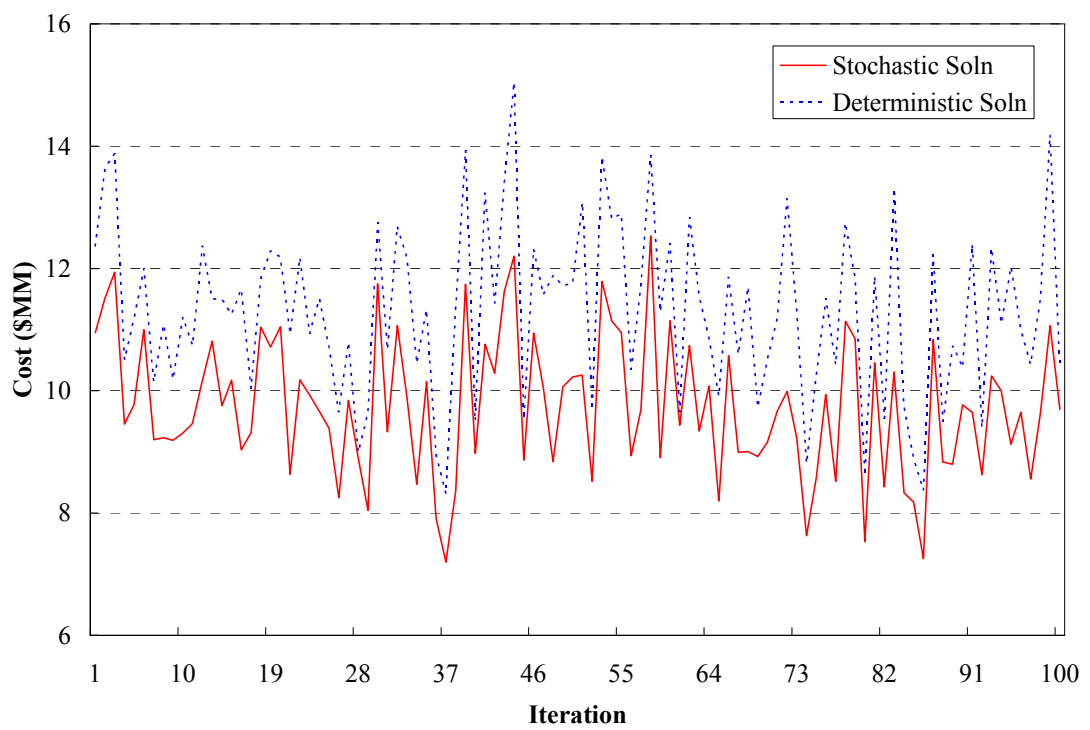

Figure 15 Simulation results for doubling uncertainty levels

\subsection{Results for Multi-cut L-shaped Method}

The size of the resulting stochastic programming is very large. As can be seen in Table 1, the size of stochastic programming model exponentially increases as the number of scenarios increases. We found that to achieve reasonable confidence intervals, we needed to use 1,000 scenarios. For the stochastic programming model with 100 scenario case, our workstation could not even generate the problem due to its huge size, i.e. the problem cannot be solved directly, although the deterministic model can be solved to optimality within one minute. By using the standard L-shaped method, we can obtain the optimal solution for the 1,000 scenario case in around 120 hours with $0.001 \%$ optimality tolerance, while the proposed multi-cut L-shaped method, requires only around 23 hours to obtain the solution with the same optimality tolerance. As can be seen in Figure 16, the standard L-shaped method requires 187 iterations to converge, while the multi-cut L-shaped method only needs 32 iterations. The advantage of multi-cut L-shaped method for this problem is assumed to be due to the master problem not requiring too much solution time compared to the one in standard L-shaped method, while the number of iterations is significantly reduced due to the "multiple" cuts.

Note that this algorithm would benefit from solving it with parallel computing, which could significantly reduce the computational times. For example, if we were to use 100 parallel CPUs, the computational time of each scenario subproblem would be at most 3 seconds in each CPU. If each CPU solves 10 subproblems in one iteration, 
after taking into account the solution time of master problem, one iteration of multi-cut L-shaped method needs at most 40 seconds. Thus, the total computational time may be reduced from around 23 hours to around 20 minutes for the 1,000 scenario instance. If we had 1,000 CPUs and allow one CPU for each scenario subproblem, the total computational time could be further reduced to around 3 minutes.

Table 1 Model size for the case problem

\begin{tabular}{lccc}
\hline \multirow{2}{*}{ Model Size } & Deterministic & \multicolumn{2}{c}{ Stochastic Programming Model } \\
\cline { 3 - 4 } & Model & 100 scenarios & 1,000 scenarios \\
\hline \# of Constraints & 6,373 & 610,374 & $6,101,280$ \\
\# of Variables & 19,225 & $1,815,816$ & $18,149,077$ \\
\# of Non-zeros & 41,899 & $4,004,697$ & $40,028,872$ \\
\hline
\end{tabular}

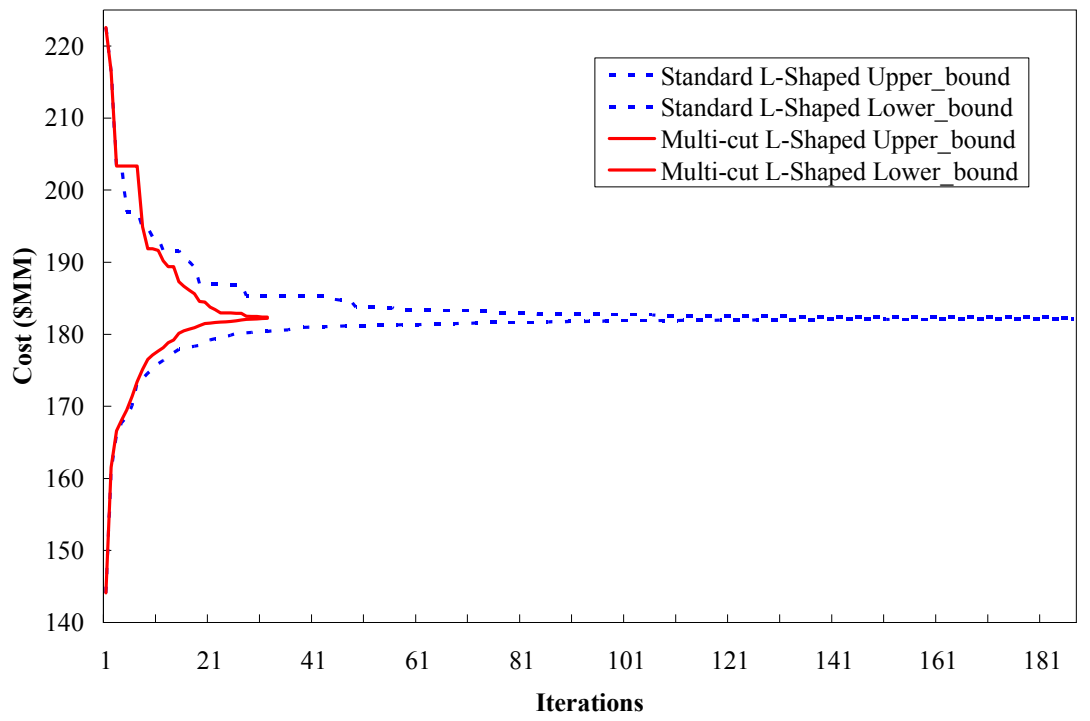

Figure 16 Comparison of two L-shaped methods

\subsection{Results for Risk Management Models}

We apply the models and algorithms for risk management discussed in Section 7.

\subsubsection{Results for managing the variance}

Figure 17 shows the statistics (mean and variance) of the total cost we obtained by managing the variance with the new objective function in (29). Here the expected (mean) cost increases as the weighted coefficient $\rho$ increases, but the variance decreases. 


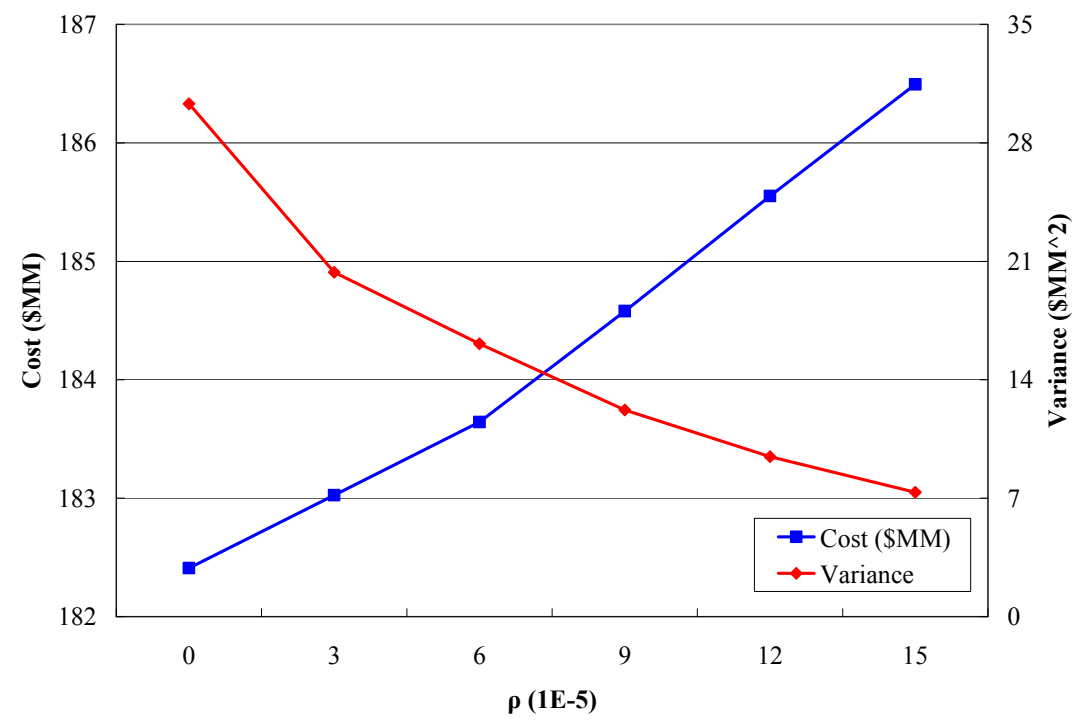

Figure 17 Efficient frontier for the variance reduction model

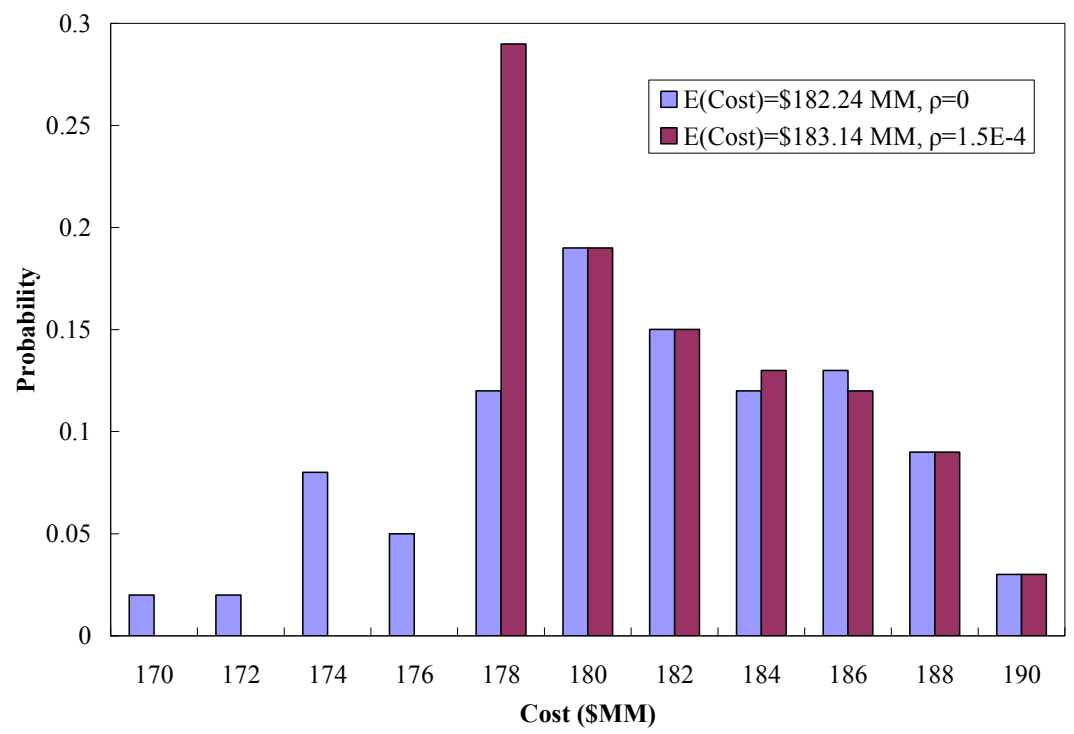

Figure 18 Histogram of the cost distribution before and after managing the variance

The histogram of cost distribution before and after managing the variance is shown in Figure 18. We can see that the spread of the expected cost is much smaller after managing the variance, but the expected cost shifts to higher costs as compared to stochastic programming. 


\subsubsection{Results for managing the variability index}

Figure 19 shows the statistics (mean and variance) of the total cost we obtained by applying the variability index model in (30) and (31). Here the expected (mean) cost increases as the weighted coefficient $\rho$ increases, but the variance decreases.

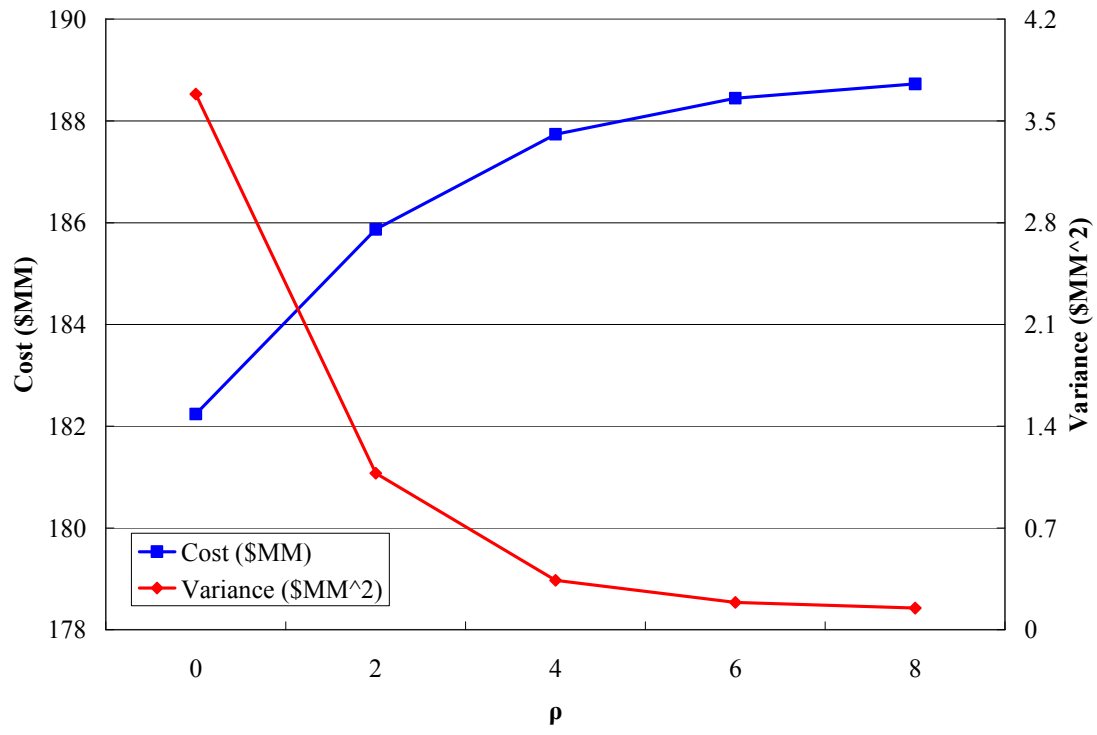

Figure 19 Efficient frontier for the variability index management model

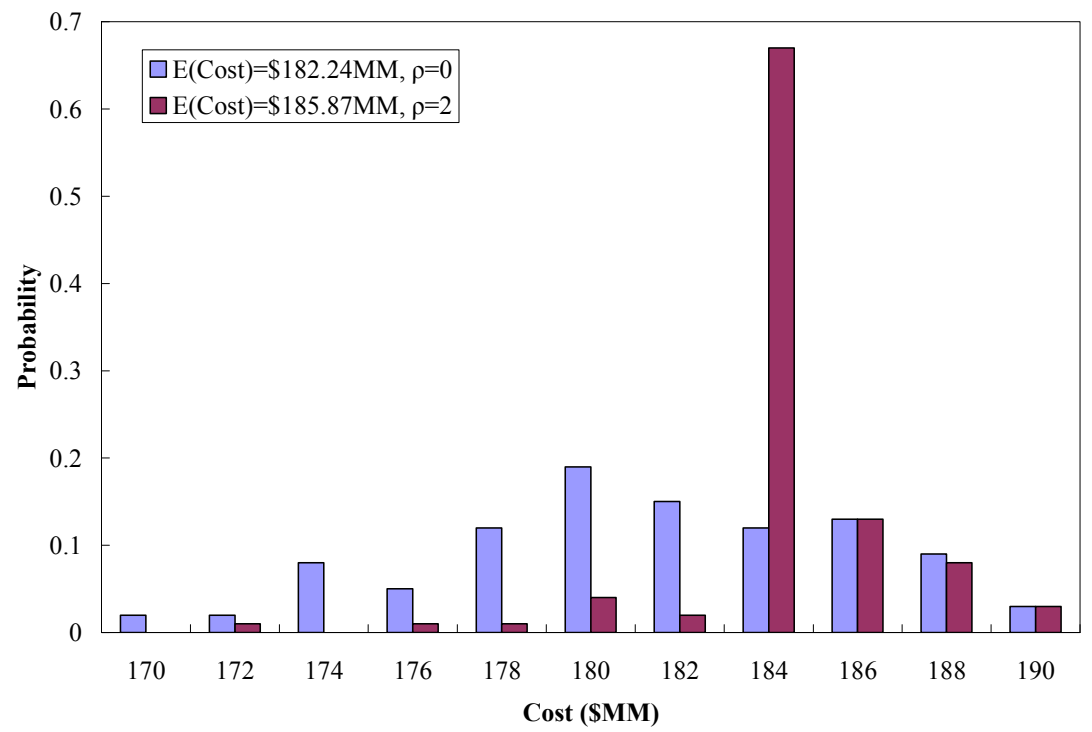

Figure 20 Histogram of the cost distribution before and after managing the variability index

The histogram of cost distribution before and after managing the variability index 
is shown in Figure 20. Again, there is a reduction in variability index of the total cost with a corresponding increase in the expected cost.

\subsubsection{Results for managing the probabilistic financial risk}

Because the probabilistic financial risk management model is a bi-criterion optimization problem, the optimal solutions yields an efficient frontier denoted as the Pareto curve, which is shown in Figure 21. Here we choose the target as \$188 MM and use 1,000 scenarios for the calculation. The comparison of cost probability distribution for the results before and after risk management is given in Figure 22. As we can see, the optimal solution of stochastic programming model has $8 \%$ probability that total cost is higher than $\$ 188 \mathrm{M}$, while after probabilistic financial risk management, the new solution has only $2 \%$ probability to have high cost (greater than $\$ 188 \mathrm{M}$ ), which means that the risk of high cost has been significantly reduced after risk management, although the expected total cost has increased.

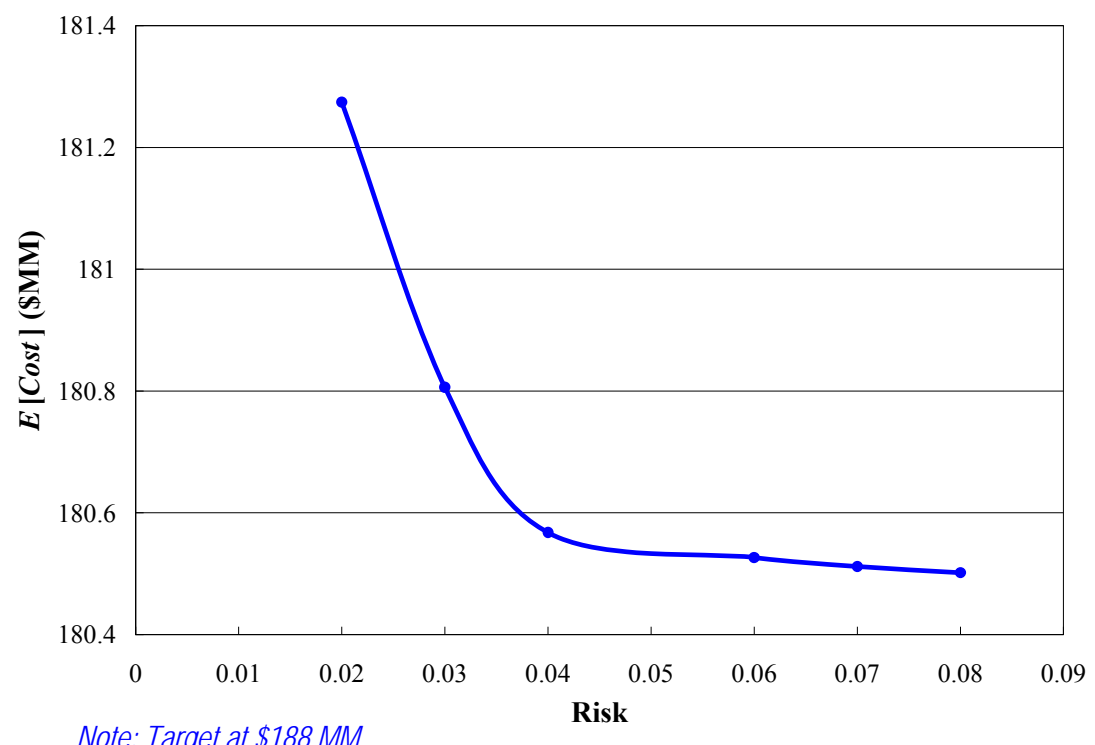

Figure 21 Pareto curve for probabilistic financial risk management model 


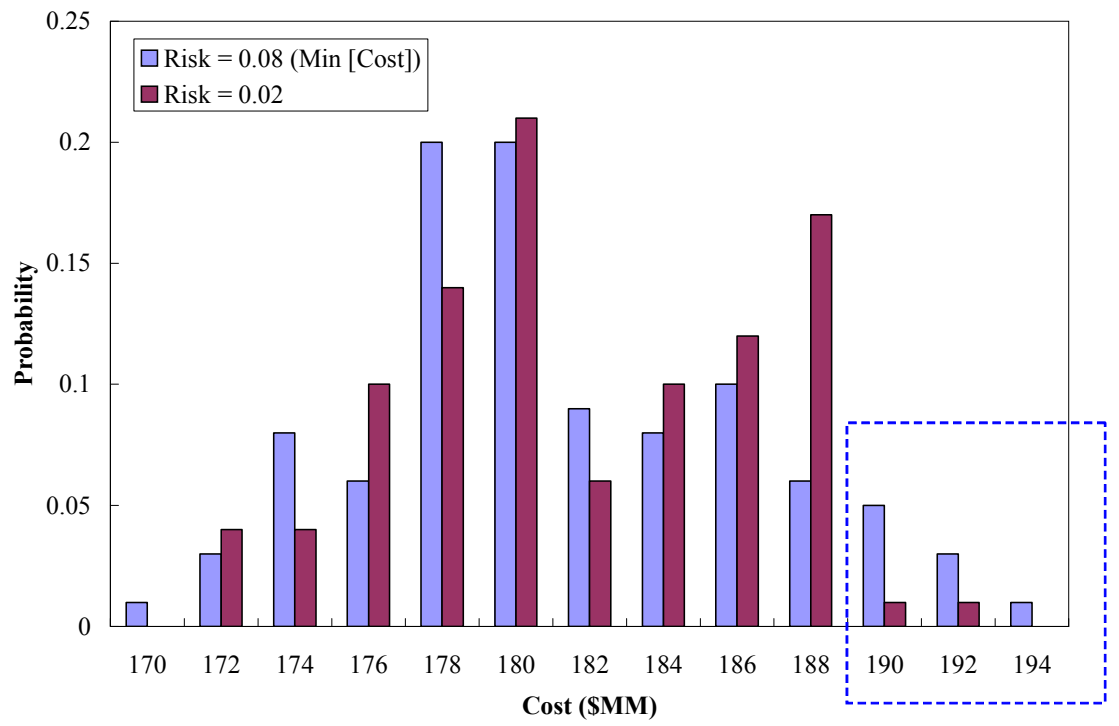

Figure 22 Comparison of the cost distribution before and after managing the probabilistic financial risk (target at $\$ 188 \mathrm{M}, 2 \%$ vs. $8 \%$ )

\subsubsection{Results for managing the downside risk}

A result after downside risk management with target at $\$ 188 \mathrm{MM}$ is given in Figure 23. Similarly we can see the risk in the high cost area has been significantly reduced, while the total expected costs have a small increase. We can also see that after downside risk management the cost has a much larger chance to be between $\$ 186 \mathrm{MM}$ and $\$ 188 \mathrm{MM}$, which is the target for downside risk management.

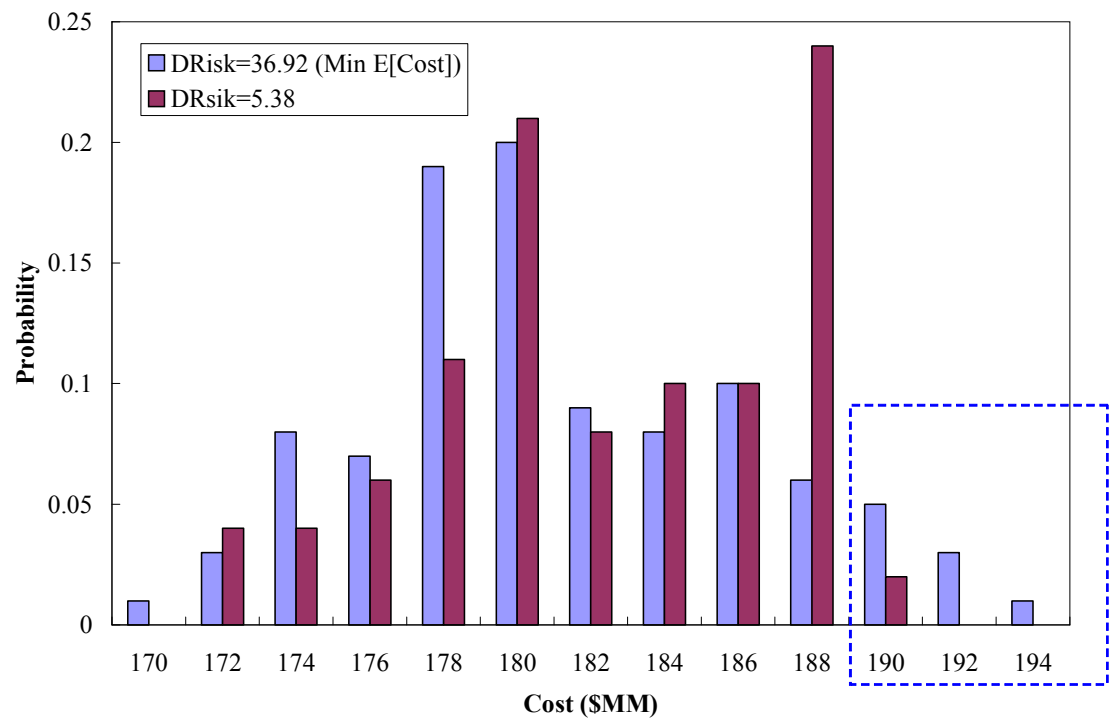

Figure 23 Comparison of the cost distribution before and after managing the downside risk (target at $\$ 188 \mathrm{MM}$ ) 


\subsubsection{Discussion}

By comparing the results for different risk management methods based on our case study, we can see that total expected cost will increase after risk management. However, probabilistic financial risk management and downside risk management are more effective in reducing the risk of high cost. Managing the variance and variability, can lead to less variance, but will usually reduce the probability of lower costs and increase the risk of higher cost due to the shift towards higher expected cost. Based on our case study, the downside risk management model appears to be the best choice because it can effectively reduce the high cost risk and is not computationally demanding compared to the probabilistic financial risk management.

\section{Conclusion}

In this work, we developed a two-stage stochastic linear programming approach for the tactical planning of a global multi-product chemical supply chain that is subjected to uncertainties in demands and freight rates. Monte Carlo sampling and the associated statistical methods are applied and incorporated into the stochastic programming model to avoid the large number of scenarios required. We also developed a simulation framework to assess the potential improvement of using stochastic programming in the supply chain planning process compared with traditional deterministic approaches. The results of our case study show that on average cost savings of $5.70 \%$ could be achieved by using the stochastic programming model on a monthly basis. To solve the large scale case study effectively, we developed a multi-cut L-shaped solution method. Computational studies show that significant savings in CPU times can be achieved by using this algorithm.

To explicitly consider the risks included in the global supply chain planning process, we studied four risk management models by using different risk measures. A real world case study was presented to demonstrate the effectiveness of the proposed models and algorithms. Computational studies suggest that probabilistic financial risk management model and downside risk management model are more effective in reducing high cost risk compared with the popular variance management and variability index management models. 


\section{Acknowledgment}

The authors gratefully acknowledge financial support from The Dow Chemical Company, the Pennsylvania Infrastructure Technology Alliance (PITA) and the National Science Foundation under Grant No. CMMI-0556090.

\section{Nomenclature}

\section{Sets/Indices}

$K \quad$ Set of facilities (including plants and distribution centers) indexed by $k$

$K_{P} \quad$ Set of manufacturing plants indexed by $k$

$K_{D C} \quad$ Set of distribution centers indexed by $k$

$R \quad$ Set of customers indexed by $r$

$J \quad$ Set of products indexed by $j$

$M \quad$ Set of transportation modes indexed by $m$

$T \quad$ Set of time periods indexed by $t$

$S \quad$ Set of scenarios indexed by $s$

Decision Variables (values: 0 to $+\infty$ )

$F_{k, k^{\prime}, j, m, t} \quad$ Inter-facility freight of product $j$ from facility $k$ to $k^{\prime}$ with mode $m$ at time period $t$

$I_{k, j, t} \quad$ Inventory level of product $j$ at facility $k$ at time period $t$

$S_{k, r, j, m, t}$ Facility-customer freight of product $j$ from facility $k$ to customer $r$ with mode $m$ at time period $t$

$W_{k, j, t} \quad$ Production amount of product $j$ at plant $k$ at time period $t, k \in K_{P}$

$F_{k, k^{\prime}, j, m, t, s}$ Inter-facility freight of product $j$ from facility $k$ to $k$ 'with mode $m$ at time period $t$ of scenario $s$

$I_{k, j, t, s} \quad$ Inventory level of product $j$ at facility $k$ at the end of time period $t$ of scenario $s$

$S_{k, r, j, m, t, s}$ Facility-customer freight of product $j$ from facility $k$ to customer $r$ with mode $m$ at time period $t$ of scenario $s$

$W_{k, j, t, s} \quad$ Production amount of product $j$ at plant $k$ at time period $t$ of scenario $s, k \in K_{P}$

$S F_{r, j, t, s} \quad$ Unmeet demand of product $j$ in customer $r$ at time period $t$ of scenario $s$

Cost $1 \quad$ First stage cost 
Cost $2_{s} \quad$ Second stage cost of scenario $s$

Cost $\quad$ Cost of scenario $s$

$E[$ Cost $] \quad$ Total expected cost

$\Delta_{s} \quad$ Upper partial mean of scenario $s$

$\psi_{s} \quad$ Positive deviation between target $\Omega$ and the cost of scenario $s$

Decision Variable (values: 0 or 1 )

$Z_{s} \quad$ Binary variable. Equal to 1 if the cost of scenario $s$ is larger than target $\Omega$

\section{Parameters}

$p_{s} \quad$ Probability of scenario $s$

$d_{r, j, t, s} \quad$ Demand of product $j$ in customer $r$ at time period $t$ of scenario $s$

$I_{k, j}^{0} \quad$ Initial inventory level of product $j$ at facility $k$

$\gamma_{k, k^{\prime}, m, j, t} \quad$ Freight rate of product $j$ from facility $k$ to $k^{\prime}$ with mode $m$ at time period $t$

$\gamma_{k, r, j, m, t} \quad$ Freight rate of product $j$ from facility $k$ to customer $r$ with mode $m$ at time period $t$

$\gamma_{k, k^{\prime}, j, m, t, s} \quad$ Freight rate of product $j$ from facility $k$ to $k^{\prime}$ with mode $m$ at time $t$ of scenario $s$

Freight rate of product $j$ from facility $k$ to customer $r$ with mode $m$ at time period $t$ of scenario $s$

$h_{k, j, t} \quad$ Unit inventory cost of product $j$ in facility $k$ at time period $t$

$\delta_{k, j, t} \quad$ Unit throughput cost of product $j$ in facility $k$ at time period $t$

$\eta_{r, j, t} \quad$ Unit penalty cost of product $j$ for lost unmeet demand in customer $r$ at time period $t$

$Q_{k, j} \quad$ Capacity of plant $k$ for product $j, k \in K_{P}$

$I_{k, j, t}^{m} \quad$ Minimum inventory of product $j$ at facility $k$ at time period $t$

$\lambda_{k, k^{\prime}, j, m} \quad$ Shipping time of product $j$ from facility $k$ to facility $k^{\prime}$ with mode $m$

$\lambda_{k, r, j, m} \quad$ Shipping time of product $j$ from facility $k$ to customer $r$ with mode $m$

$\Omega \quad$ Target for risk management 


\section{Reference}

1. Grossmann IE. Enterprise-wide Optimization: A New Frontier in Process Systems Engineering. AIChE Journal. 2005;51:1846-1857.

2. Shah N. Process industry supply chains: Advances and challenges. Computers \& Chemical Engineering. 2005;29:1225-1235.

3. Chopra S, Meindl P. Supply Chain Management: Strategy, Planning and Operation. Saddle River, NJ: Prentice Hall; 2003.

4. Shapiro JF. Modeling the supply chain. Pacific Grove, CA: Duxbury Press; 2001.

5. Wilkinson SJ, Cortier A, Shah N, Pantelides CC. Integrated production and distributioin scheduling on a Europe-wide basis. Computers \& Chemical Engineering. 1996;20:S1275.

6. Bok J-K, Grossmann IE, Park S. Supply Chain Optimization in Continuous Flexible Process Networks. Industrial \& Engineering Chemistry Research. 2000;39(5):1279-1290.

7. Jackson JR, Grossmann IE. Temporal decomposition scheme for nonlinear multisite production planning and distribution models. Industrial \& Engineering Chemistry Research. 2003;42(13):3045-3055.

8. Chen C-L, Lee W-C. Multi-objective optimization of multi-echelon supply chain networks with uncertain product demands and prices. Computers \& Chemical Engineering. 2004;28:1131-1144.

9. Oh HC, Karimi IA. Global multiproduct production-distribution planning with duty drawbacks. AIChE Journal. 2004;50(5):963-989.

10. Guillen G, Badell M, Espuna A, Puigjaner L. Simultaneous optimization of process operations enhance the integrated planning/scheduling of and financial decisions to chemical supply chains. Computers \& Chemical Engineering. 2006;30(3):421-436.

11. Subrahmanyam S, Pekny JF, Reklaitis GV. Design of Batch Chemical Plants under Market Uncertainty. Industrial \& Engineering Chemistry Research. 1994;33:2688-2701.

12. Wellons HS, Reklaitis GV. The design of multiproduct batch plants under uncertainty with staged expansion. Computers \& Chemical Engineering. 1989;13:11.

13. Petkov SB, Maranas CD. Design of Single Product Campaign Batch Plants under Demand Uncertainty. AIChE Journal. 1998;44:896.

14. Gupta A, Maranas CD. Managing demand uncertainty in supply chain planning. Computers \& Chemical Engineering. 2003;27:1219-1227.

15. Gupta A, Maranas CD, McDonald CM. Mid-term supply chain planning under demand uncertainty: customer demand satisfaction and inventory management. Computers \& Chemical Engineering. 2000;24:2613-2621.

16. You F, Grossmann IE. Optimal Design and Operational Planning of Responsive Process Supply Chains. In: Papageorgiou, Georgiadis, eds. Process System Engineering: Volume 3: Supply Chain Optimization. Weinheim: Wiley-VCH; 2007:107-134.

17. You F, Grossmann IE. Design Responsive Process Supply Chains under Demand Uncertainty. Submitted to Computers \& Chemical Engineering; 2007.

18. Liu ML, Sahinidis NV. Optimization of Process Planning Under Uncertainty. Industrial \& Engineering Chemistry Research. 1996;35:4154.

19. Shapiro A. Stochastic programming by Monte Carlo simulation methods. 
Stochastic Programming E-Prints Series. 2000:03.

20. Shapiro A, Homem-de-Mello T. A simulation-based approach to two-stage stochastic programming with recourse. Mathematical Programming. 1998;81(3):301-325.

21. Mak W-K, Morton DP, Wood RK. Monte Carlo bounding techniques for determining solution quality in stochastic programs. Operations Research Letters. 1999;24:47-56.

22. Eppen GD, Martin RK. A Scenario Approach to Capacity Planning. Operations Research. 1989;37(4):517-527.

23. Mulvey JM, Vanderbei RJ, Zenios SA. Robust Optimization of Large-Scale Systems. Operations Research. 1995;43(2):264-281.

24. Ahmed S, Sahinidis NV. Robust Process Planning Under Uncertainty. Industrial \& Engineering Chemistry Research. 1998;37(5):1883-1892.

25. Applequist GE, Pekny JF, Reklaitis GV. Risk and uncertainty in managing chemical manufacturing supply chains. Computers \& Chemical Engineering, 2000;24(9-10):2211-2222.

26. Barbaro AF, Bagajewicz M. Managing Financial Risk in Planning under Uncertainty. AIChE Journal. 2004;50(5):963-989.

27. Bonfill A, Bagajewicz M, Espunia A, Puigjaner L. Risk Management in Scheduling of Batch Plants under Uncertain Market Demand. Industrial \& Engineering Chemistry Research. 2004;43:741-750.

28. Pongsakdi A, Rangsunvigit P, Siemanonda K, Bagajewicz MJ. Financial risk management in the planning of refinery operations. International Journal of Production Economics. 2004;103:64-86.

29. Guillen G, Bagajewicz M, Sequeira SE, Espuna A, Puigjaner L. Management of Pricing Policies and Financial Risk as a Key Element for Short Term Scheduling Optimization. Industrial \& Engineering Chemistry Research. 2005;44(3):557-575.

30. Birge JR, Louveaux F. Introduction to Stochastic Programming. New York: Springer-Verlag; 1997.

31. Yi G, Reklaitis GV. Optimal Desing of Batch-Storage Network Considering Exchange Rates and Taxes. AIChE Journal. 2007;53(5):1211-1231.

32. Van Slyke RM, Wets R. L-Shaped Linear Programs with Applications to Optimal Control and Stochastic Programming. SIAM Journal on Applied Mathematics. 1969;17(4):638-663.

33. Balas E. Disjunctive Programming and a Hierarchy of Relaxations for Discrete Continuous Optimization Problems. SIAM Journal on Algebraic and Discrete Methods. 1985;6(3):466-486.

34. Sawaragi Y, Nakayama H, Tanino T. Theory of Multiobjective Optimization. Orlando, Florida: Academic Press; 1985.

35. Dua V, Pistikopoulos EN. Parametric Optimization in Process Systems Engineering: Theory and Algorithms. Proceeding of Indian National Science Academy. 2004;69(A):429-444.

36. Brooke A, Kendrick D, Meeraus A, Raman R. GAMS- A User's Manual: GAMS Development Corp.; 1998. 